A POLARIZED BEAM FOR THE M-3 LINE

D. Underwood, E. Colton, H. Halpern, G. Theodosiou, A. Yokosawa, G. Shapi ro, S. Gray, A. Beretvas, H. Mietenin, T. Mulera, J. Roberts

\title{
MASTER
}

NoThe, ALN ONLY

PRTIONG AFTHS REPORT ARE ILLEGIBLE It

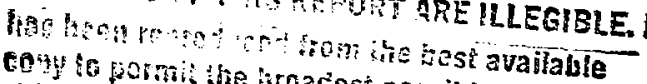
abitity.

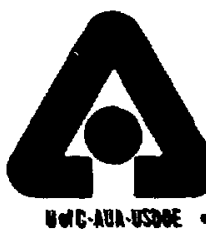




\section{A POLARIZED BEAM FOR THE M-3 LINE*}

D. Underwood, E. Coltcn, H. Halpern, G. Theodosiou, and A. Yokosawa Argonne National Laboratory

Argonne, Alinois 60439

G. Shapiro

Lawrence Berkeley Laboratory

University of California

S. Gray

University of Indiana

Bloomington, Indiana

A. Beretvas

Fermilab

Batavia, Illinois

H. Mietenin, T. Mulera, J. Roberts

Rice University

Houston, Texas

\section{ABSTRACT}

We propose a beamline for polarized protons to be built in the M-3 line of the Meson Laboratory utilizing lambda decays. This beamline would provide a clean source of polarized protons or an enriched beam of antiprotons or polarized antiprotons.

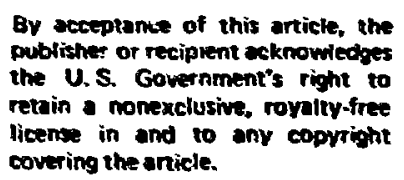

*Work performed under the auspices of the United States Energy Research and Development Administration. 
CONTENTS

Page

I. Introduction . . . . . . . . . . . . . . . . . . 1

II. Some Methods of Producing Polarized Beams

A) Summary of the Argonne Symposium of June 10, 1977. 2 (The End-Point Scheme)

B) The new polarization method . . . . . . . . . . 8 (The Transverse Scheme)

III. Transport of a Polarized Beam. . . . . . . . . . . . 13

IV. Polarization Reversal . . . . . . . . . . . . . . . . 19

V. Polarimeter . . . . . . . . . . . . . . . . . 26

VI. Beam Design . . . . . . . . . . . . . . . . . . 27

VII. Intensities . . . . . . . . . . . . . . . . . . . 35

VIII, Targeting and Sweeping . . . . . . . . . . . . . . . . 41

IX. Modifications to Meson Lab . . . . . . . . . . . . . . 46
A) Target Train
B) M-3 Line
C) Other Considerations

x. Magnets Needed . . . . . . . . . . . . . . . 


\section{INTFODUCTION}

We propose a beamine for polarized protons to be built in the $M-3$ line of Meson Lab. Utilizing lambda decays, thiss beamline would provide a clean source of polarized protons from 50 to $350 \mathrm{GeV} / \mathrm{c}$ with $400 \mathrm{GeV} / \mathrm{c}$ primary beam. It would also provide an enriched beam of antiprotons or polarized antiprotons at momenta above $50 \mathrm{GeV} / \mathrm{c}$. The $\mathrm{M}-\mathrm{S}$ line was chosen because the beam can be built with no net bend in the transport from primary target to experimental target, this condition being necessary to avoid depolarizing the beam. Forward production of lambdas is also necessary for high rates. The physics to be done in this beam is described in a separate propasal. 


\section{NETHODS OF PFODUCING POLARIZZED BEAMS}

A) Surmary of the Argonne Symposium of June 10, 1977

The polarized beam from the argonne zOS is very good in terms of flivx, emittance, and low background, but to go to higher energies we should consider other approaches. All the higher energy acceleraturs are strong focusing machines and it now appears that cue todepolarizing rescnances acceleration of polarized beams will be difficult. Also, operation of these accelerators in a polarized beam mode may not be attempted for some time because of interference with other programs. We would also like a polarized antiproton beam.

For the production of palarized secondary beams, we can consider strong, electromagnetic, and meak effecis. There was a proposal by J. Basen et al., to produce a polarised neutron bean in the M-3 line using the Schwinger polarization effect. This involves multiple electromagnetic scattering through the neutron mgnetic moment. One might get sizable polarization and moderate beam intensities.

A similar geometry could be used to select neutrons or protons polarized through the strong interaction. Protons produced at siall angles in a diffraction dissociation process such as $\mathrm{nA} \rightarrow \mathrm{Ap} \pi^{-}$may be polarized at some energies and angles. Unfortunately, there is no data on polarization in diffraction dissociation at the moment. 
We now consider: weak interactions.

The protons from lambria decay are polarized along thcir direction of motion in tize lambda rest frame. This polarization has been measured by Cronin and Overseth and others to dutermine the relative amoumis of $s$ wave and $p$ wave in the decay. For unpolarized lambalas; the polarization of the proton in the direction of the proton is $a=.65$, where

$$
\alpha=\frac{2 R e\left|s p^{*}\right|}{|s|^{2}+|p|^{2}}
$$

$s$ and $p$ are the $s$ wave and $p$ wave amplitudes in the parity violating decay.

The problem in bulding a polarized beam is to tag this decay direction in the lambda rest frame.

Under transformation froind dre c.m. system to the lab system, the spin vector of the proton remains almost fixed, as shown in Figure ${ }^{1}$. One can tag the direction of muximum polarization of the proton in various ways in experiments.

One method was discussed with respect to the Fermilab neutral hyperon beam by Overseth and Heller. A slightly different scheme was proposed in a paper by Overseth and sandweiss. 2 This involved a complete kinematic reconstruction of each proton interacion and the lambda decay which produced the proton. 
All schemes involve knowing the proton direction with respect to the Iambda, but we can deal with much higher rates if the direction can be chosen in the laboratory frame by beam geometry or some other parameter which does not require kinematic reconstruction of the Iambda decays. In particular, we do not want to deal with every decay since only a small fraction of the final proton interactions will be interesting.

Three schemes has been considered at the time of the June loth Symposium 3 wich involve either no explicit reconstruction or a simple correlation. The first of these has been discussed in a CERN letter of intent by Fidecaro et al. By collimating the lambda beam down to limit the production $p_{1}$ distribution, one can choose the magnitude and direction of $p_{1}$ of the proton with respect to the lambda by choosing the proton beam direction with respect to the collimated lambda beam direction. This scheme produces a transversly-polarized beam, ano it is not difficult to change polarization direction (e.g. up, down on alternating accelerator spilis). The intensity is very limited, however, because most lambdas are lost by the severe collimation. This inherent limitation is due to the fact that the proton has only $.1 \mathrm{GeV} / \mathrm{c}$ momentum in the lambda rest frame, while typically $\left\langle p_{1}>\right.$ for lambda production is $.5 \mathrm{GeV} / \mathrm{c}$. Representative distributions are shown in Fig. 3. This method has been superseded by a method to be described later ("new transverse scheme"). The second scheme involves a pion momentum tag for a fixed (narrow band) proton momentum. The pion momentum from a lambda decay will tag the proton direction and thus the polarization. The momentum of the lambda must be known, as it will be for 
given pion and proton momentum. As an example, with beam line transporting $p$ or $\overline{\mathrm{p}}$ at $85 \mathrm{GeV} / \mathrm{c} \pm 5 \%, 7 \mathrm{GeV} / \mathrm{c}$ pions correspond to decays with the proton forward in the lambda c.m., and $24 \mathrm{GeV} / \mathrm{c}$ pions correspond to backward decays.

This tagging scheme has a severe limitation in that the $\pi^{+}$ flux to be tagged will be at least a factor of 10 more intense than the $\bar{p}$ beam. For simple time correlation tagging, this limits a $\overrightarrow{\mathrm{p}}$ beam to about $10^{5}$ second. With elaborate phase space tagging one might do better, but background problems in tagging telescopes near the $\bar{n}$ production target could be severe.

The third scheme is very attractive for producing highmomentum polarized protons. The method has been studied by $\mathrm{CERN}^{5}$ for an existing beam line and by a CERN-ORSAY collaboration. The lambda flux falls off sharply at large $x$ as shown in Fig. 2 . By chonsing fast protons from lambda decays, we will obtain protons primarily from forward decaying events. The resulting proton polarization will be longitudinal. The advantages of this scheme are 1) large fluxes, 2) no tagging, 3) low backgrounds, 4) less critical beam dump and sweeping requirements than for tagging schemes. Unfortunately its application to $\bar{p}$ beams seems to be limited to polarization of a few percent at $|x|<.1$. Relevant distributions for this scheme are shown in Figs. 3, 4, 5, anc : 


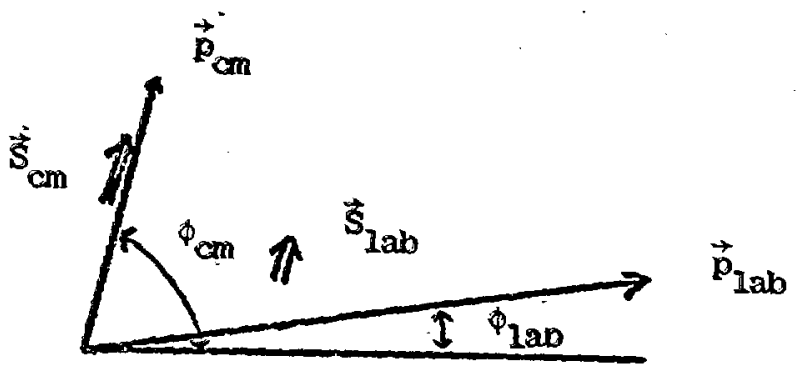

Fig. 1 Spin transformation to laboratery frame.

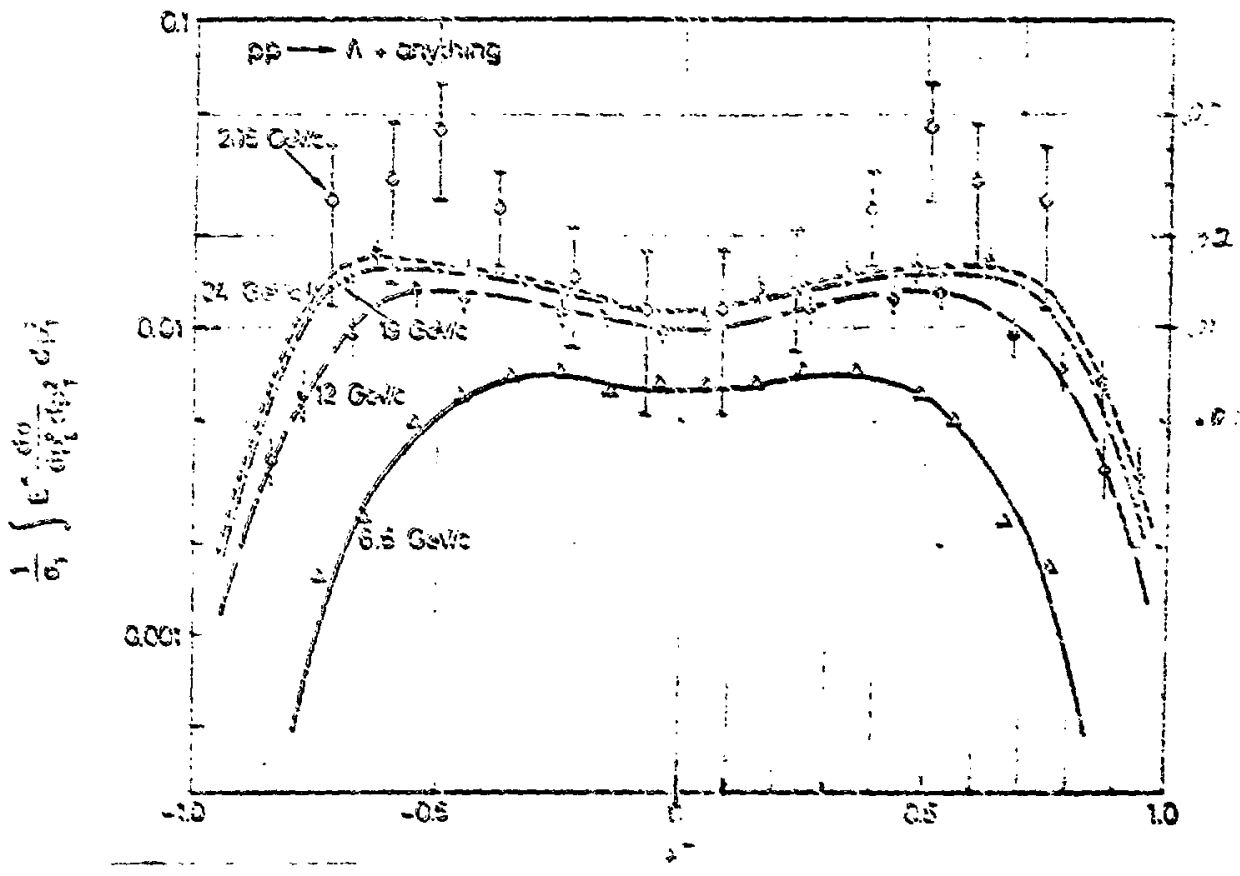

Fig. 2 Inclusive distributions for $A$ obtained in pp collisions between 7 and $200 \mathrm{GeV}$. 
Fig. 3

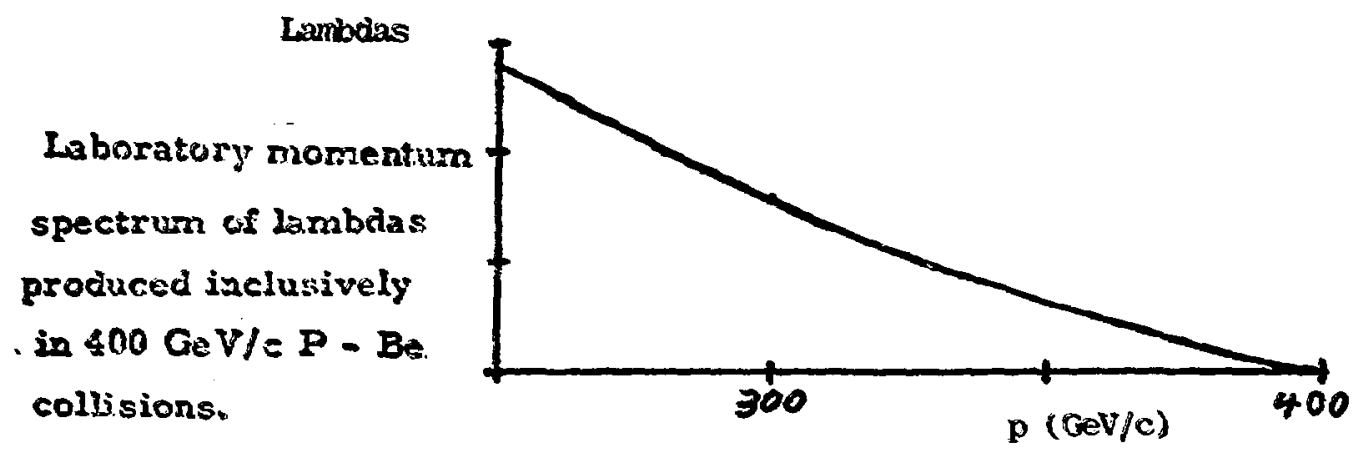

Fig. 4

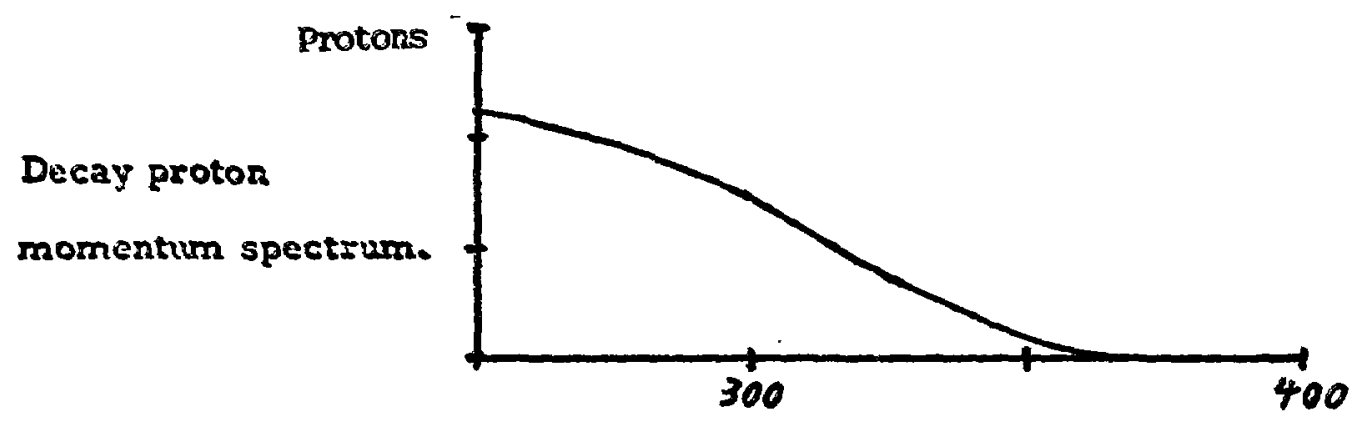

Fig. 5

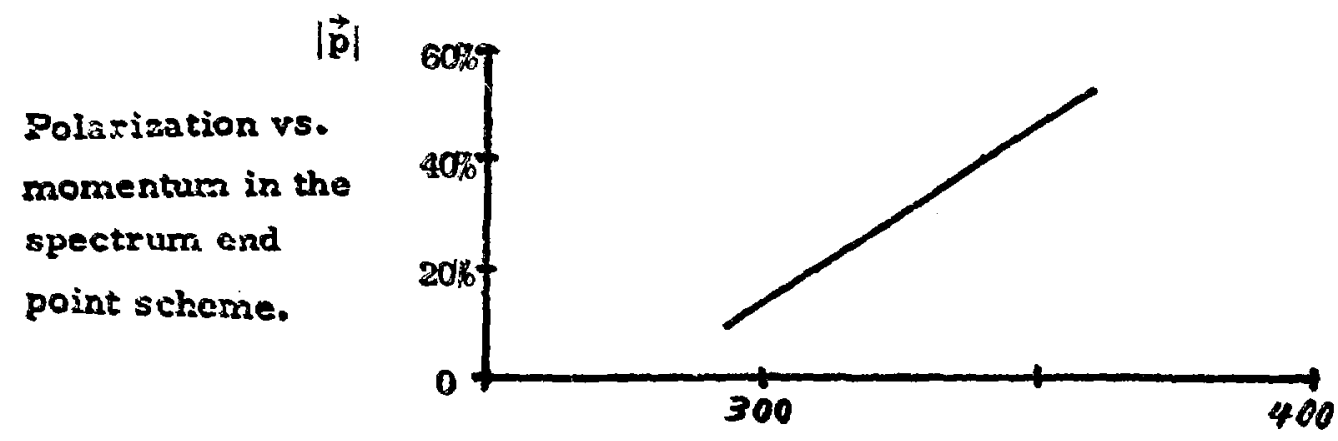

Fig. 6

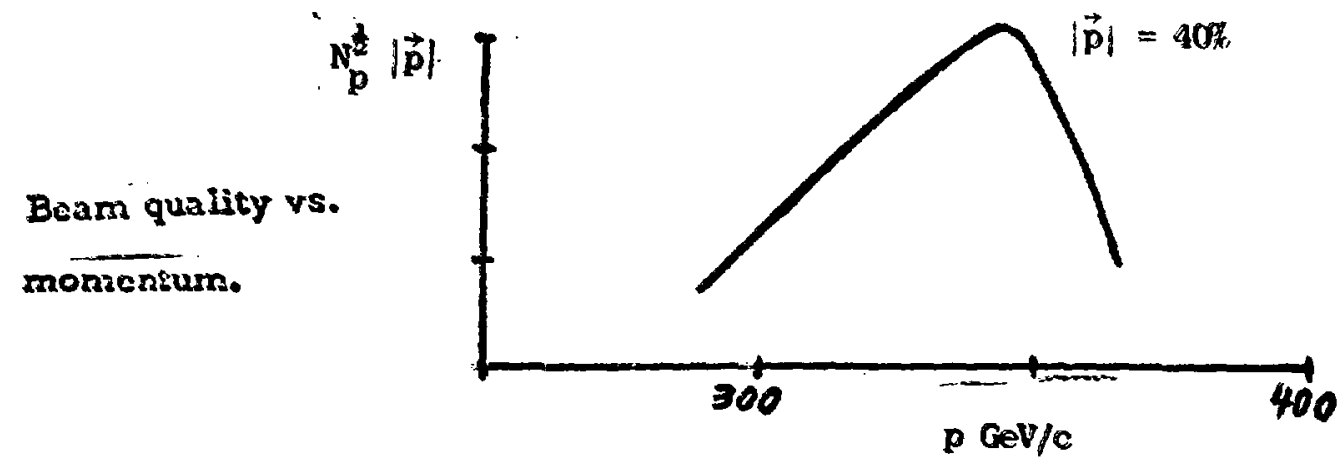


B. THE NEN POLARIZATION SCHEME

In any experiment with a polarized beam, it is essential to reverse the polarization from spill to spill to cancel systematic errors to first order.

In the course of rtudying polarized-proton beams from lambda decay, it was realized that there is a reasonably straightforward way to select two different polarization directions of a fermion coming from an unpolarized parent particle in a parity violating decay.

There are actually two ways to do this, one of which has been known for some time. The older method typically produces beams two orders of magnitude less intense than those available with the new method for protons.

It will illustrate the methods with the case of ${\Lambda^{\circ} \rightarrow p}^{-}$.

The basic idea is that polarization direction corresponds to decay directio: of the $p$ in the $\Lambda^{0}$ rest frame. If we can select this decay direction in the lab Irame with some property of the beam line, we have selected the polarization. The old way to do this was to collimate the beam of parent particles $\left(\Lambda^{0}\right)$ tightly before the decay, so that the decay momentum (Q value) of the decay product (P) could be observed. With the coming off transverse to the $\Lambda^{\circ}$ with 100 , MeV/c momentum, we must collimate the $\Lambda^{\circ}$ beam to less than 100. HeV/c transverse momentum spread. This old method is very inefficient because the average $p_{\perp}$ for $\Lambda^{\circ}$ production is $500 \mathrm{MeV} / \mathrm{C}$ and we can accept only a tiny portion of the total $\Lambda^{\circ}$ production. 
The new method works as follows: consider lambdas decaying at some distance from the production target. For transverse decays (near $90^{\circ}$ in $\Lambda^{\circ}$ c.m.) extrapolation of the proton track back to the region of the production target will give a transverse position correlated with the direction of the transverse decay. The effective (not real) beam spot at the target will have a position-polarization correlation like this:

Fig. 7

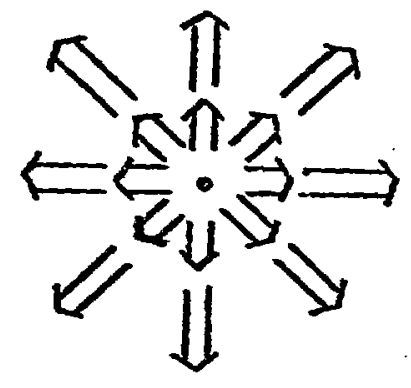

Where the center of the effective beam spot corresponds to longitudinal decays ( $P$ at $0^{\circ}$ or $180^{\circ}$ in $\Lambda^{\circ}$ c.m.). The trick in actually producing a polarized beam lies in selecting segments of this effective beam spot at the first focus. With a sliding collimator or two moving collimators one can select polarization on a spill-to-spill basis.

One can think of the new scheme as transforming away the spatial effects of the production $p_{1}$ so that there is no need to collimate the production $p_{1}$. As shown in Fig. 8, protons coming from decays exactly along the lambda direction will be focused to a point at the first focus. The remaining spatial distribution is due only to the $p_{1}$ of the protons with respect to the lambdas and not to the production $p_{1}$ of the lambdas. 

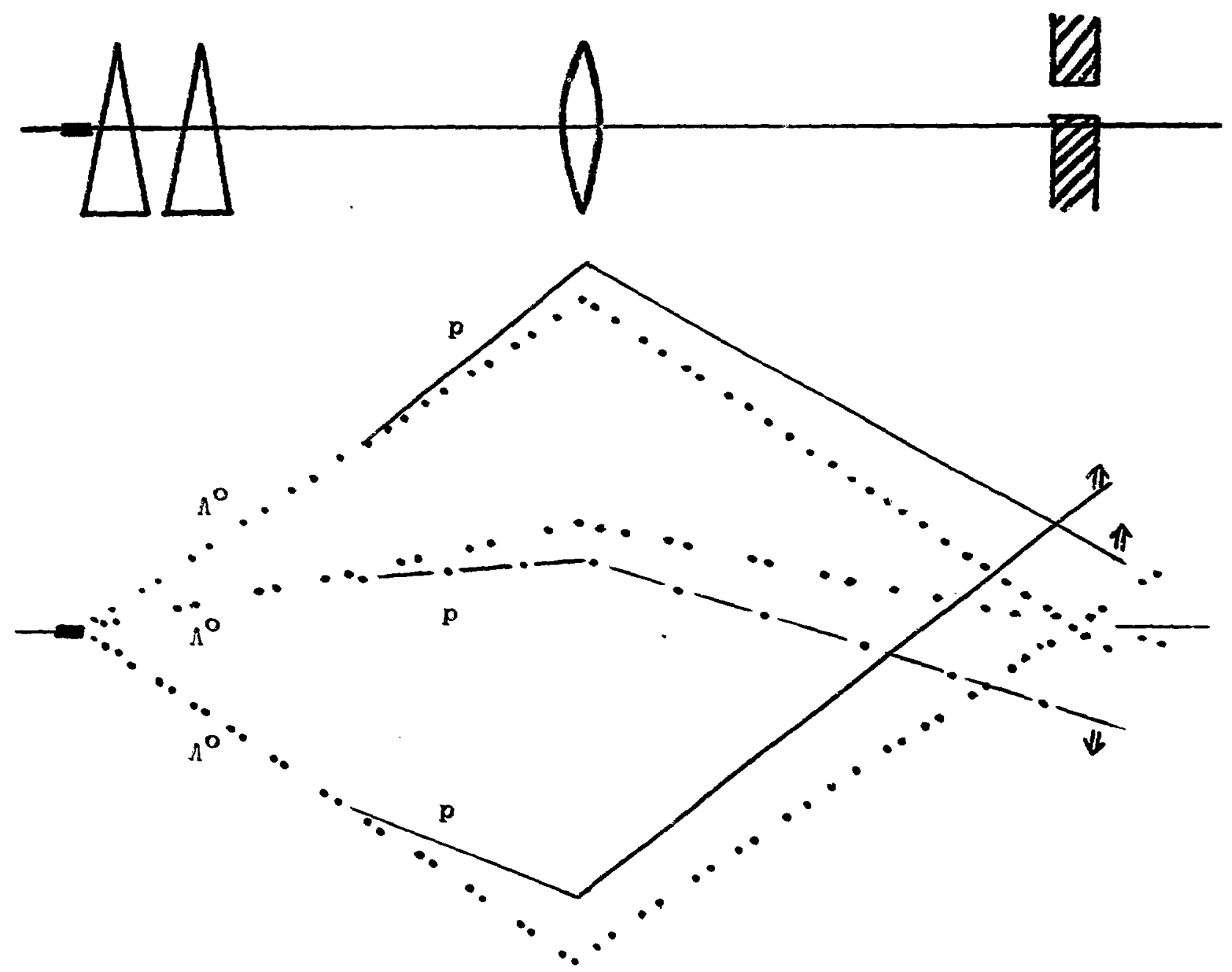

Fig. 8 Polarization method (collimation off-center at a focus). 
Integral Curves

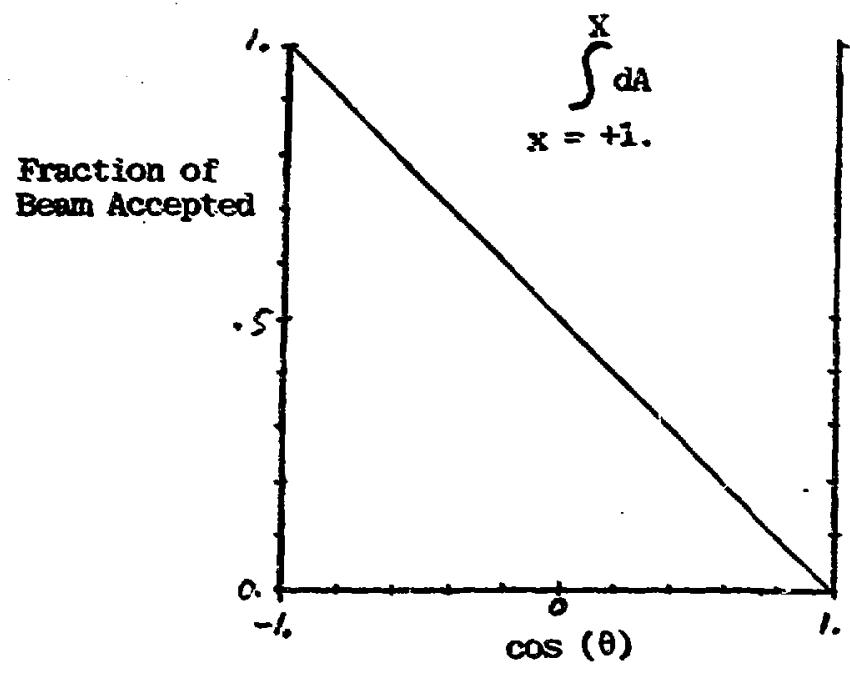

Differential Curves
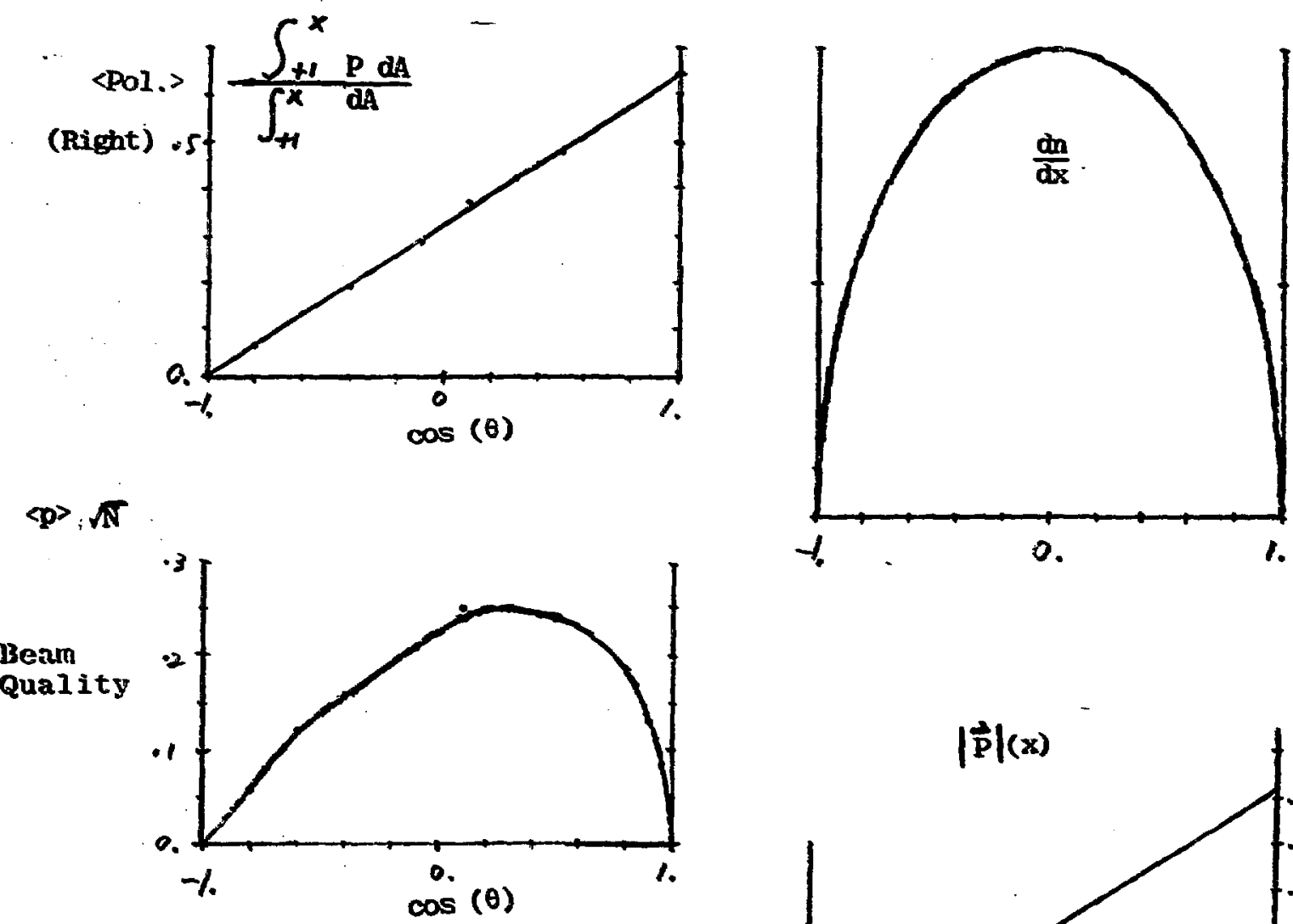

Fig. 9 Polarization and intensity curves for the transverse scheme. (Idealized to point production target and single momentim.) 
In implementing this scheme in a beam line there are a number of complications and factors to be considered.

I) We obtain high rates by accepting large production $P_{1}$ for the lambdas. We save a large divergence beam.

ii) It is not trivial to transport a large divergence proton beam without depolaxizing it with strong quadrupoles. These transport problems will be described in the beam transport section, but it is worth noting here that useful polarization only exists at even numbered foci although the collimation may be done at any early focus.

iii) The beam must be momentum selected to some extent, and the correlation between polarization and phase space is lost completely in the plane of bending if the momentum spectrum is roughly flat. The polarization collimator must be at $90^{\circ}$ to the momentum selection collimator.

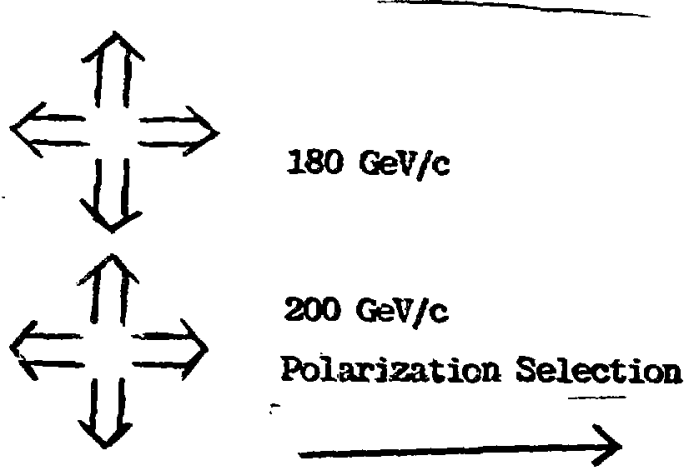

Mamentum

Selection

Fig. 10 
With respect to the schemes discussed in the June 10 Sjmposium this new transverse scheme: 1) has higher intensity; 2) is far more practical for polarized antiprotons; 3) el iminates the need for frontporch operation to get various beam energies for total cross-section experiments 4) allows polarization reversal with less disturbance of the beam. The disadvantages are: 1) the maximum momentum is about $300 \mathrm{GeV} / \mathrm{C}$ for $400 \mathrm{GeV} / \mathrm{C}$ incident; 2) there may be pion contamination of a few percent at lower energies which would not be present with the longitudinal scheme and front-porch operation. 


\section{TRANSPORT OF A POLARIZED BEAM}

It was clear sore time ago that a focusing element or set of elements such as a quadrupole triplet would depolarize a high divergence beam such as we were proposing. The first order solution is to use another identical element after a focus to compensate and return spins to their original directions

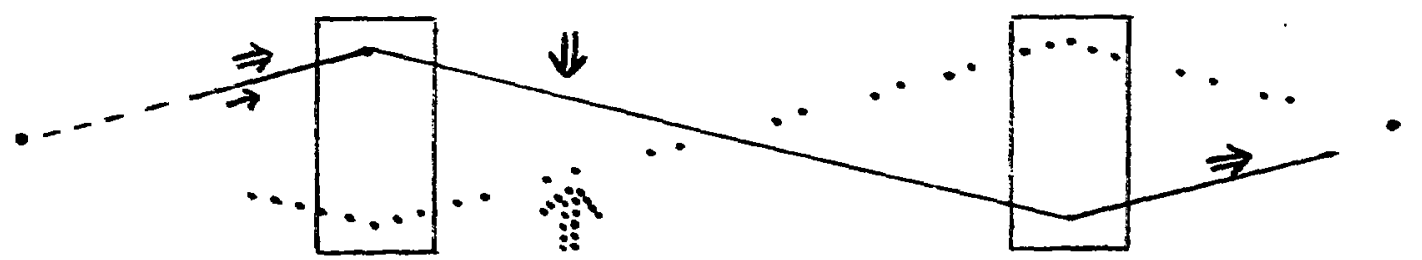

Quadrupole Triplet
Quadrupole Triplet

Fig. 11 Spin precession in quadrupoles for high-momentum large divergence beam.

In the limited case illustrated, this means a transfer matrix of $\left(\begin{array}{ll}1 & 0 \\ 1 & 1 \\ \frac{1}{1}\end{array}\right)$ after two stages. If we use this as only an overall constraint for the beam 1 ine, we must be very careful about finite spot size and finite momentum bite effects in intermediate stages. The $\left(\begin{array}{cc}1 & 0 \\ \frac{1}{f} & 1\end{array}\right)$ constraint requires that the initial focusing stage near the production target match the final focusing stage near the experimental target. There are further requirements, such as there being an odd number of foci between the first and last stage. 
By pencil and paper ray tracing one can find the approximate field integra: involved in depolarization on paths from an offaxis beam spot. This is a second order effect in a beam line where the first order (beam spot center) effects have been eliminated by use of a $\left(\begin{array}{cc}1 & 0 \\ \frac{1}{f} & 1\end{array}\right)$ transfer. For two stages of $1: 1$ transport with 25, m from focus to lens this would be about $9 \mathrm{kG}-\mathrm{m}$ for a $0.5 \mathrm{~cm}$ displacement or a spin rotation of about $30^{\circ}$.

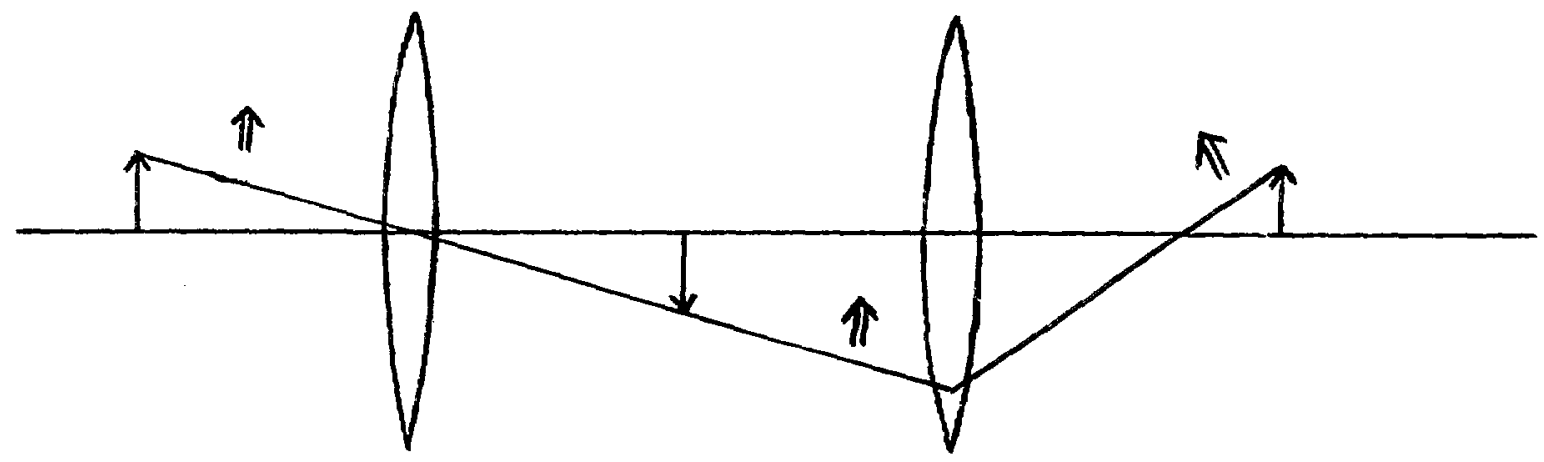

Fig. 12 Spin precession on rays originating away from the beam spot center in a beam with no precession for rays originating on the optical axis.

This effect and similar effects go away completely for a beam with a $\left(\begin{array}{ll}1 & 0 \\ 0 & 1\end{array}\right)$ transfer matrix as shown in Fig. 13. 

We have designed for an overali transfer matrix of $\left(\begin{array}{ll}1 & 0 \\ 0 & 1\end{array}\right)$
in each dimension.

$a_{11}$ is spacial magnification

$a_{22}$ is angular magnification

${ }^{a} 12$ is the dependence of final position on initial angle ( 0 at a focus)

$a_{21}$ is the inverse of the effective focal length of the lens system ( 0 for infinite focal length)

Demanding that $a_{11}$ and $a_{22}$ be 1 , insures that rays originating at the center of the target (and ending at the center of the focus) have zero field integral in the quadrupoles and no spin precession. Demanding that $a_{12}$ be 0 is asking for a focus at the experinental target.

If $a_{21}$ is zero, parallel rays are transformed to parallel rays and the field integral (spin precession) for rays at the edge of the beam spot is zero.

The particular beam design found has a transfer matrix at the first focus of $\left(\begin{array}{ll}a & 0 \\ 0 & \frac{1}{\alpha}\end{array}\right)$ and a matrix of $\left(\begin{array}{ll}1 & 0 \\ 0 & 1\end{array}\right)$ at the final focus.

The design of each half of the beam is similar to that arrived at by Christian Huygens around the year 1680. He designed a system to eliminate lateral chromatic effects in simple lens systems analogous to quadrupole triplets. The beam design is a stretched version of his eyepiece design. We have the element spacing equal to the sum of the focal lengths rather than the .5 to .7 times the sum used in telescopes. 


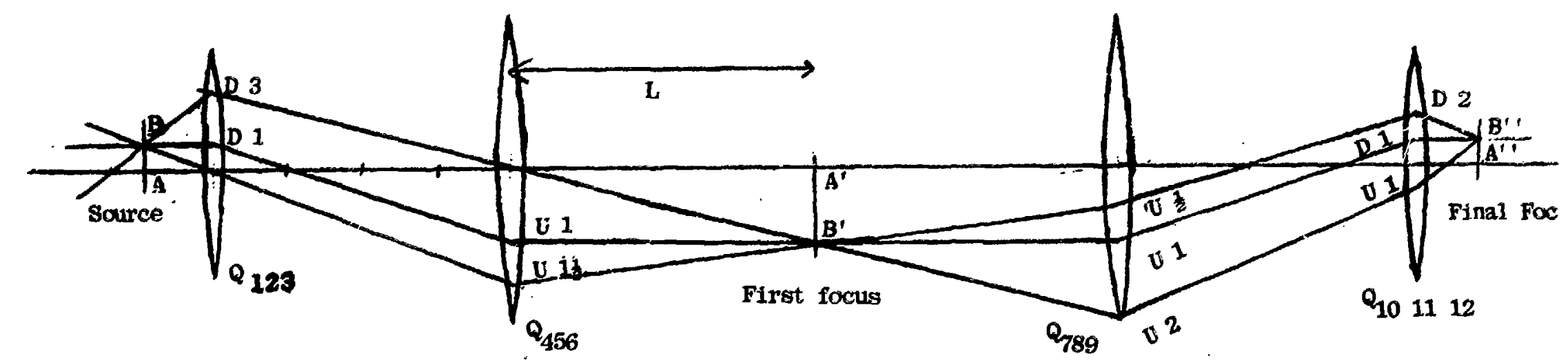

Fig. 13

Diagram showing that there is no net spin precession for any off-axds or largo divergence ray. This alagrem is for a bean with ${ }_{0}^{1} 0$ transfor matrix, synmetrically placed elements with spacing $L$, and Idealized thin quadrupoles. The strength of $Q_{123}$ is three times that of $2_{456}$. The labels "D 1 ", "U 12/", etc. refer to one unit of downward-bending field integral, one and one-half units of upward-bending field integral, etc. 
In addition to these one-dimensional considerations in each dimension, one must also take into account the non-commuting nature of spin precessions in orthogonal directions. We are constrained to compensate all bends between sets of quadrupoles becauae of this non-commutativity. We are further constrained to compensate all momentum dispersion between quadrupole sets if the beam is to transport a large momentura bite for high iutensity. Thus the only viable combination of bends and quads is that shown below. (This can be in a focusing or parallel section.)

Fig. 14

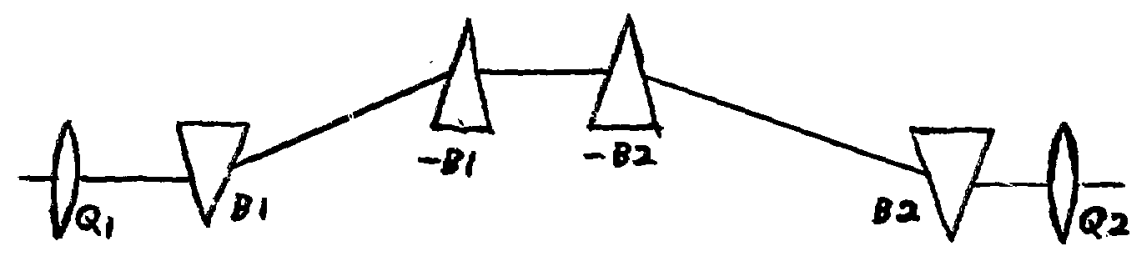

Aside from these large-scale constraints in first and second order, one might expect local depolarization effects in a set of quadrupoles. For quadrupole triplets the non-commutativity effects do not exist to first order as shown below. They have to be considerea further in high order.

Fig. 15
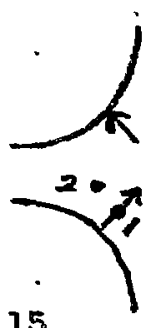

$Q_{1}$
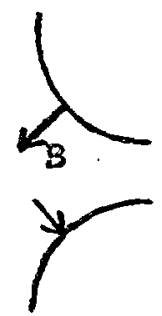

1

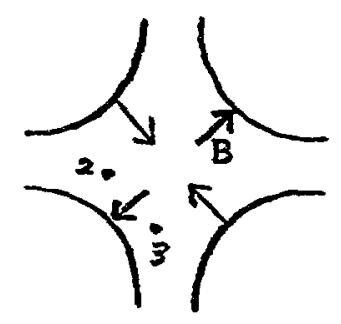

$Q_{2}$

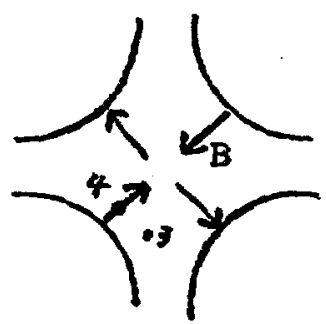

$\mathbf{Q}_{3}$

For small excursions within the quadrupoles the beam bending is almost in a plane. 
The polarization direction is actually not quite constant in transforming from the lambda c.m. to the laboratory. In the convention of Bargmann, Micbel and Telegdi where the spin is represented by a 4-vector, it is $S \cdot V$ which is constant, where $V$ is the 4-velocity. For transverse decays $S \cdot V=0$ and there is an effective rotation of the spin direction of about 6 degrees forward in the laboratory frame. There is a formula for this rotation given by Dalpiaz, Jansen and Coignet: 6

$$
\tan \left(\theta_{\mathrm{Lab}}\right)=\sin \left(\theta_{\mathrm{cm}}\right) /\left[\lambda_{\mathrm{cm}}\left(\cos \left(\theta_{\mathrm{cm}}\right)+\beta_{\mathrm{cm}} / \beta_{\mathrm{Lab}}\right)\right]
$$

This rotation is still about 6 degrees in the nucleon-nucleon center-of-mass in the experimental target. The resulting longitudinal component of polarization is probably negligible in most transverse-polarization experiments, but could be removed with the spin precession magnets. 


\section{POLARIZATION REVERSAL}

With proton beams, spin reversal can be done with spin precession. magnets, utilizing the large anomalous magnetic moment of the proton.

One development in polarization reversal schemes is the recognition that it is possible to use a set of reversing magnets in such a way that there is no motion of the beam in the beam transport system or at the experiment. The setup is a variant of the $E-B I$ scheme or Siberian snake which makes more extensive use of the fact that orthogonal $90^{\circ}$ rotations do not commute. The scheme uses 8 magnets of $27.4 \mathrm{kG}-\mathrm{m}$ field integral each. It can flip the polarization of a longitudinally polarized beam with no external motion of the beam. It can also translate a fixed horizontal polarization into spin-to-spill alternating vertical polarization. These are among the polarization directions to be dealt with in the beam design to be described later.

One constraint on this system is the fact that the distance from the final quadrupoles to the experiment is determined by lambda production dynamics since the beam must be mirror symmetric (at least the first and last stages). This means that at the energies of interest and with the constraints of the $M-3$ line, roughly 20 . $m$ is available for the spin precession magnets. The spin precession must be done in the region of an even numbered focus, leaving the area just upstream of the experiment as the only practical place. With this constraint we can contemplate using 2 . m long magnets at $13.7 \mathrm{kG}$, or $1.52 \mathrm{~m}$ long magnets at 18 . $\mathrm{kG}$.

It is useful to note that in these schemes the beam trajectory is a segmented helix. It may be possible to come up with a twomagnet version of all this using solenoids cocked at come angle to the beam. (Ferhaps $45^{\circ}$ ?)

The 8 magnet scheme is probably the best option for end point production and experiments using longitudinal beam. It may not be necessary for experiments using the lower energy beam. 
Fig. 20 SPIN FLIPPING WITH NO NET BEAM MOTION (Transverse) (Reverse 4 of 8 Mrgnets) (No verniers)
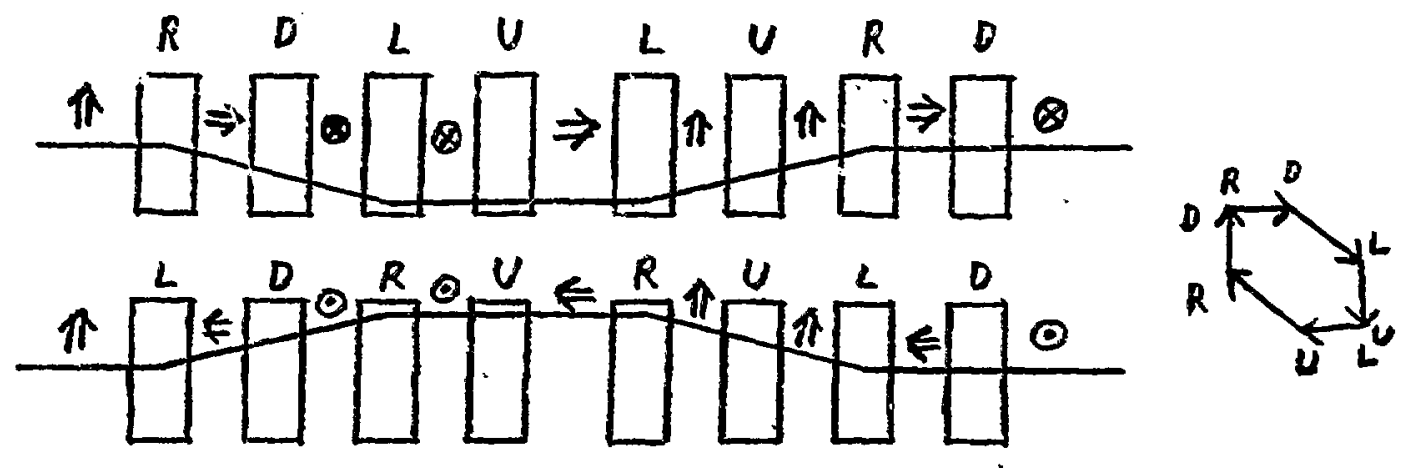

Transverse in - Transverse out
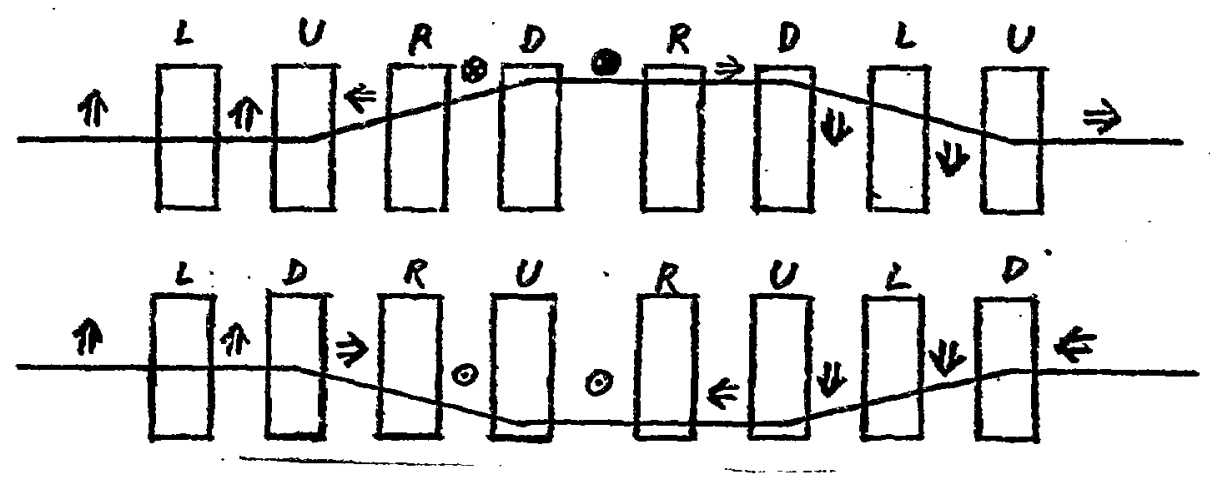

Transverse in - Longitudinal out
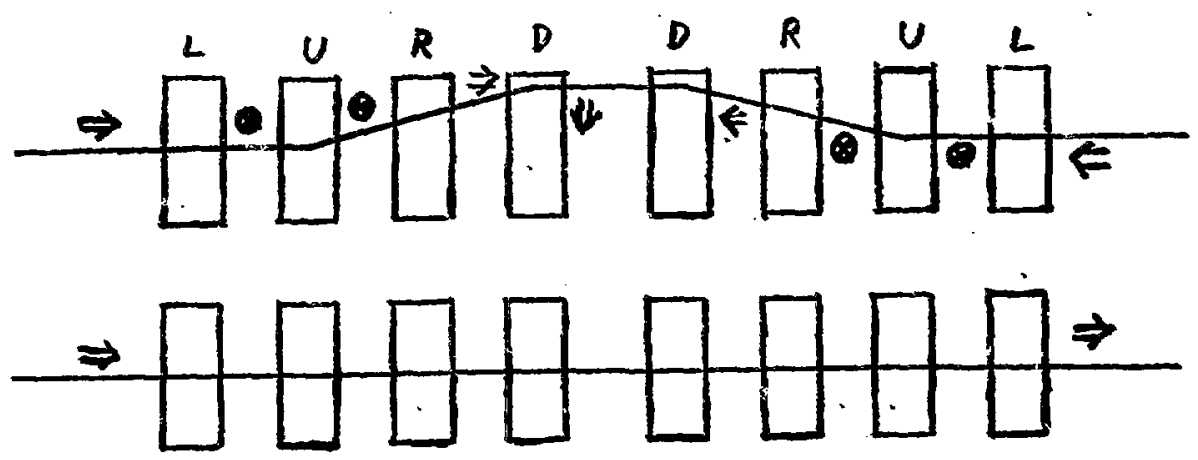

Longitudinal in - Longitudinal out 
The new scheme for obtaining polarization by collimating at a focus (transverse scheme) would require motions of the beam of only a few centimeters at any energy. (Assuming that polarization reversal were done with moving collimators - even this becomes unnecessary in a new scheme to be described, but let us discuss it here anyway. ) The changing position of the collimator hole would be compensated at some point in the beam by a small vernier magnet. Beam motion in the quadrupoles could be zero for some beam designs.

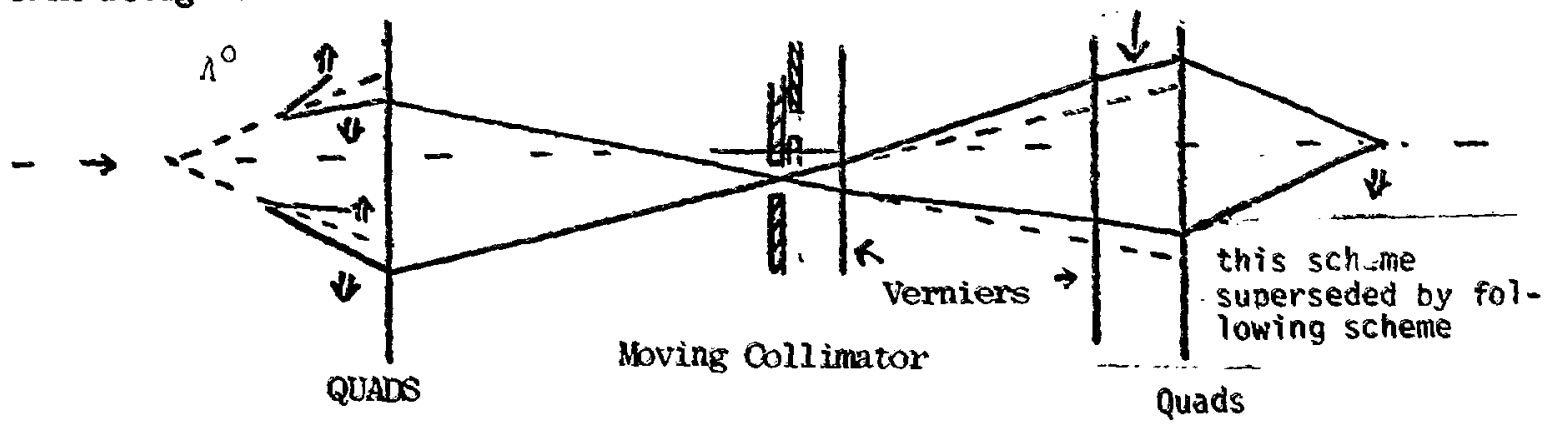

If a beam is built with a long distance from the focus to the quadrupoles this will probably be the best scheme at energies lower than the end point.

There is a trick dependent on the optics of an actual beam design which is a great improvement upon even this scheme. For a beam line consisting of two telescopic segments, one vernier in the upstream segment (upstream of the collimator at a telescopic focus) can deflect the beam in conjunction with the quadrupoles to move the spot at a fixed collimator. The beam stays parallel to the original beam axis. The only problem may be small chromatic effects (no dispersion to first order). The beam would be unchanged downstream of the collimator while changing polarization from spill to spill. 

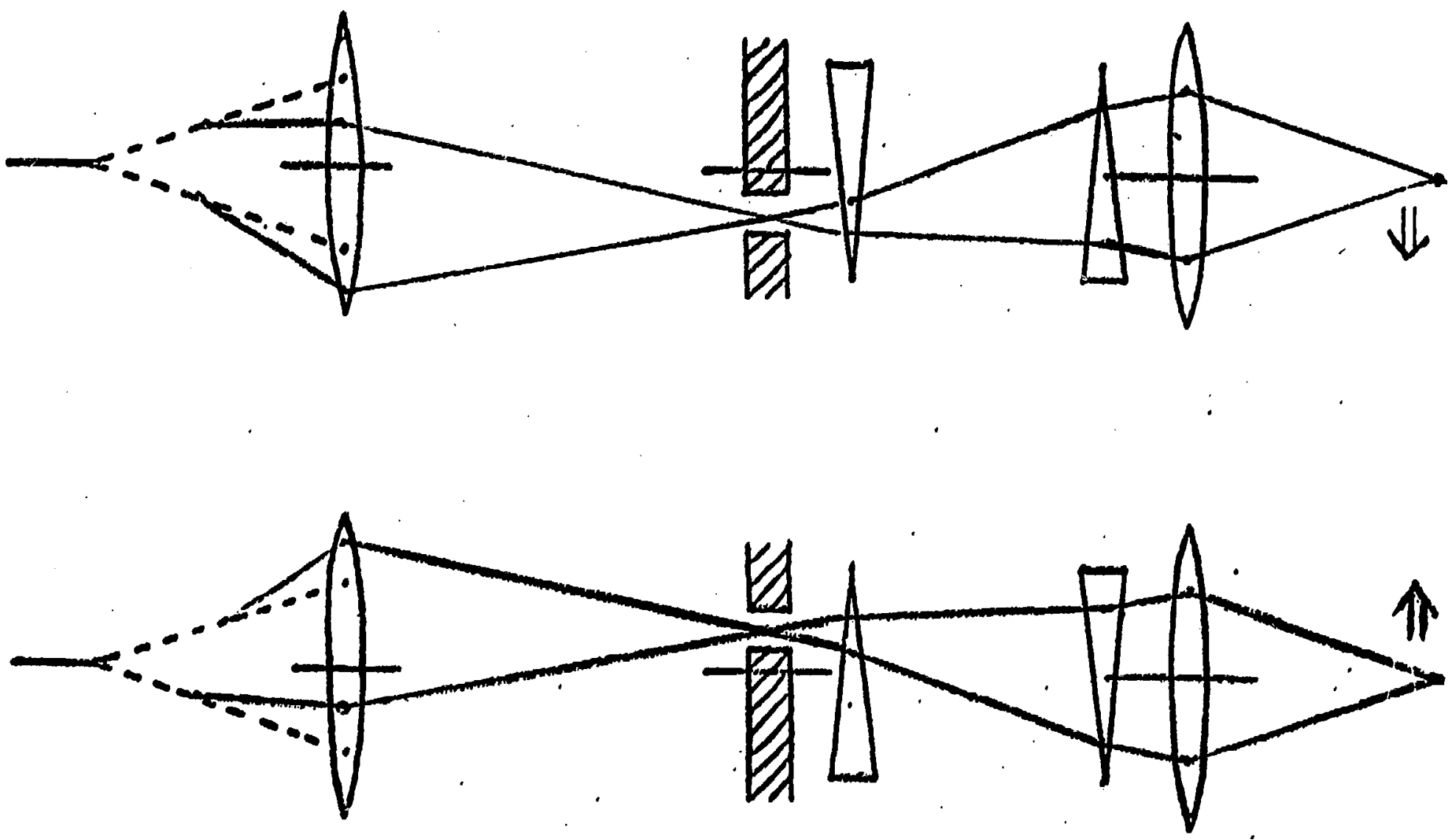

Fig. 18

Two-vernier polarization reversal.

This figure shows the verniers downstream of a mving collimator. An improved version would have one or two verniers upstream of a fixed collimator. 

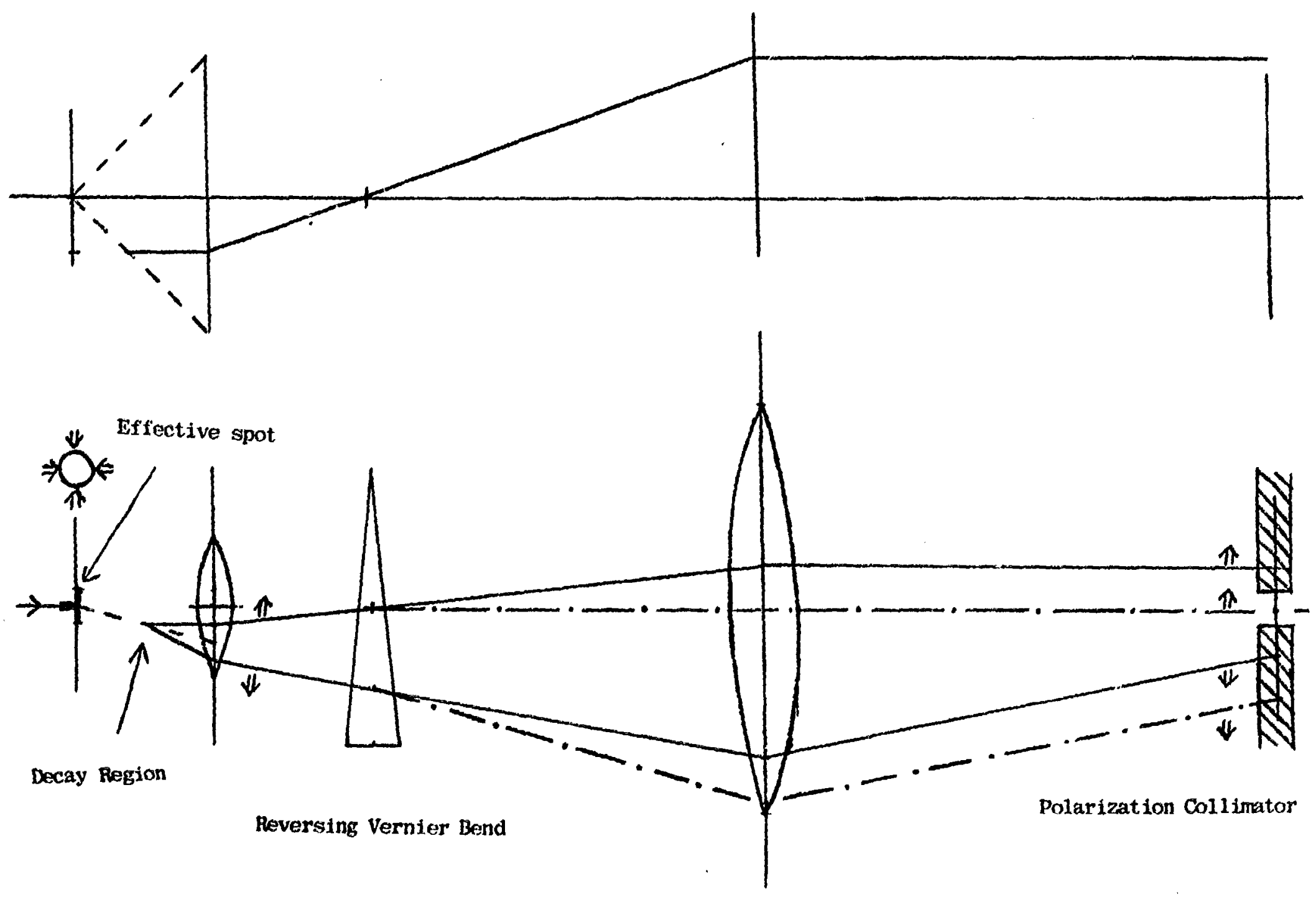

Fig. 19 - One - Vernier Polarization Selection 
VII. FOLARIMETER

While the polarization of the beam can probably be calculated to better than three per cent, including correlations of polarization with position and angle, such a calculation assumes that all magnets are operating at correct field values and all elements are surveyed in place perfectly. A bean polarimeter could greatly reduce the risk of taking data when the beam was not working properly. Even a polarization measurement only once during a data run could make the difference between a successful experiment and an unsuccessful one. Polarization correlations with position and angle within the beam spot should be ineasured as a cross check of bean performance.

Two types of polarimeters are known which could in principle give an absolute measurement of polarization. One using the Primakov effect and one using Coulomb-nuclear interference.

Relative polarization monitors using inclusive processes might be simpler to build and use but would have to be calibrated somehow. Practical details are discussed in references 5,9 and 10 .

Measurement of decays of forward produced lambdas may not be appropriate as discussed in note $D$. 


\section{BEAM DESIGN}

We have tried to investigate the beam design with several simultaneous interrelated approaches. These include:

i) Thought experiments

ii) Pencil and paper ray tracing to investigate field integral contributions which could depolarize the beam.

iii) Use of the matrix program IRANSPORT to obtain exact beam parameters for the $\left(\begin{array}{ll}1 & 0 \\ 0 & 1\end{array}\right)$ transfer matrix and to investigate the consequences of various designs.

iv) Use of the Monte Carlo program TURTLE to realistically investigate the effects of dispersion and collimators.

v) A more detailed Monte Carlo to investigate spin precession in detail. (This has not progressed too far since we deceided to deal with first order effects first).

On the basis of these considerations we have come up with one explicit design.

A beam design with a $\left(\begin{array}{ll}1 & 0 \\ 0 & 1\end{array}\right)$ transfer matrix in each dimension has been found using program TRANSPORT. There are eight constraints to be satisfied in the matrix fitting. In the best working design found so far there are eight variables. Four of these variables are quadrupole strengths, and four are dimensions built into the element placement. This is a triplet solution which is almost identical in horizontal and vertical dimensions. The doublet solution found was unsatisfactory in terms of second order chromatic effects, beam excursions within the doublets leading to depolarization, and acceptance. 
The use of quadrupole triplets is actually preferable in terms of rate and focusing for polarization selecion. The only possible problem would be in the higher field integrals required with triplets.

Once the basic design had been found with TRANSPOFT, the properties were investigated in more detail with the ray tracing program TURTLE.

With TURTLE, one can put in the effects of collimators on large momentum bites and check the second order transport calculations. Distributions were histogrammed instead of dealing with the boundaries of phase space.

The decision to plan this beam for the $\mathrm{s}^{-3}$ line which has no bend was necessary in terms of rate and flexibility. It now appears possible to get a polarized beam in the line using the transverse or collimation scheme, but the intensity would be lower by perhaps two orders of magnitude due to the necessity of limiting the phase space volume to prevent depolarization by mixed and uncompensated bends and quadrupoles. What appear to be the remaining problems in the $1-3$ design are due to the lack of bend and/or the size of the burried vacuum pipe.

With the fresent design, the beam is bent up and then down again (or perhaps left-right) in two places. First, to dump the neutral beam, the proton beam must go $\left(\begin{array}{c}\text { down } \\ \text { up }\end{array}\right)$ and start $\left(\begin{array}{c}\text { up } \\ \text { down }\end{array}\right)$ again in the front end hall. In the 400 foot a rea the beam goes through a bend to horizontal, a quadrupole triplet, and another bend for momentum selection. Bends and collimators for momentum selection and polarization selection are in the 600 foot area. The compensating bend is in the 1000 foot area.

The beam pipe is only 12 . inches in diameter and displacements of the beam of roughly a foot (within \pm a factor of 2 ) are needed both for neutral beam dumping and momentum selection. 


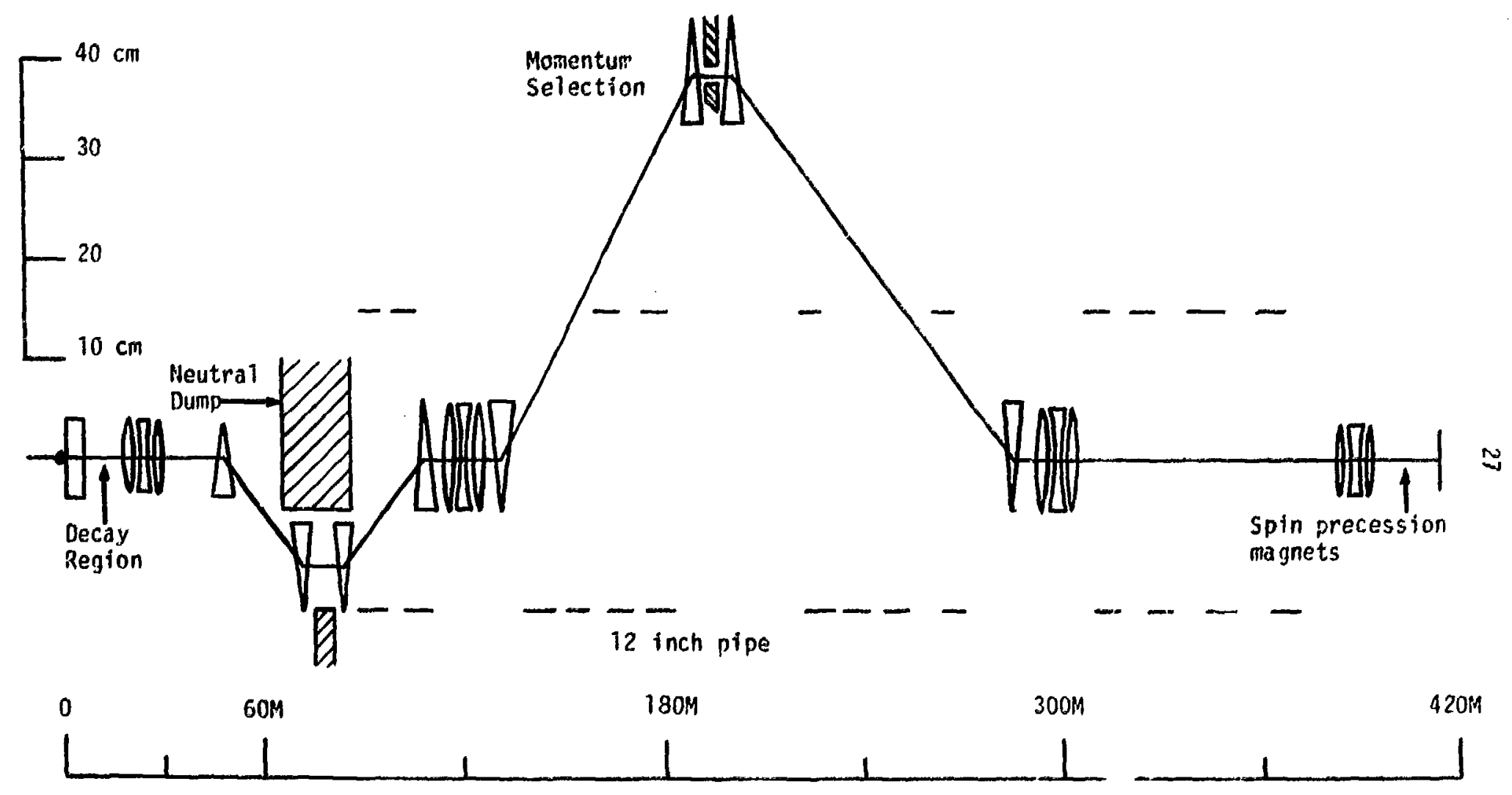

Fig. 21 Sketch of beam line 

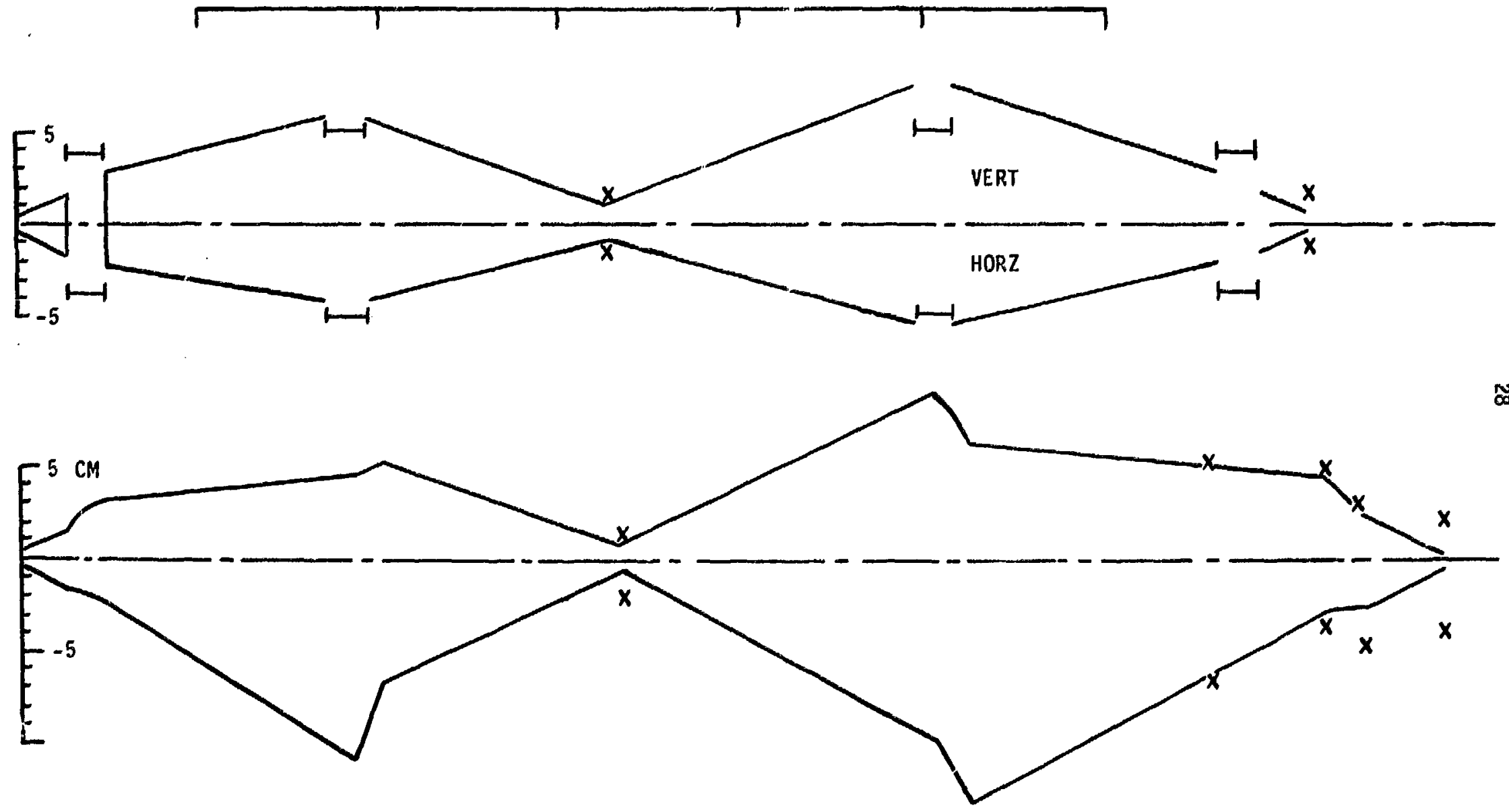

Fig. 22 Beam envelopes for triplet version and doublet version. The crosses $(x)$ show the effect of a $\pm 5 \%$ momentum bite. The doublet version is clearly not acceptable in terms of polarization selection or spot size at the final focus. 
The present design requires only conventional iron magnet technology. The constraints on magnet sizes come primarily from the layout of the M-3 line and the proximity of nearby beams. The $M-1$ and $M-6$ beams may originate from targets only four to eight inches from the M-2/M-3 target if a three-way split is built for meson $1 \mathrm{ab}$.

The halls available for magnets in the 11-3 line are only fifty feet long. The simplest design would require fourty feet of four-inch aperture quadrupoles plus two bends of six feet each in one of these halls (450' area).

The eight magnet polarization reversal scheme requires eight magnets of $27.4 \mathrm{kG}-\mathrm{m}$ field integral and rather large aperture. The aperture is determined by the lowest momentum at which the magnets would be used in a reversing mode. At $340 \mathrm{GeV} / \mathrm{c}$ where this is the best reversal scheme, we need roughly $13 \mathrm{~cm}\left(5^{\prime \prime}\right)$ aperture. At $50 \mathrm{GeV} / \mathrm{c} 42 \mathrm{~cm}$ would be required but this is probably neither practical nor desirable since the reversal scheme available for low momentum would require only two small reversing verniers in the beam line. The eight magnets would probably require at least 15 . $M$ in the beam. Some of the optimum TRANSPORT solutions give 26. $m$ from the final quads to the experimental target. 
Using TURTI.E, have obtained histograms of momentum acceptance and spot size at the polarization collimator for displacements of the beam of 6", 12", and 24" at the momentum collimator for a reasonable collimator size. The collimator size chosen was 2 . $\mathrm{cm}$ since the edge of the phase space boundary was \pm 1 . $\mathrm{cm}$ at the momentum focus at $340 \mathrm{GeV} / \mathrm{c}$. Spot size is inversely porportional to momentum. The spot size was obtained by using a point production target and "lambda decays" (rms additions to divergence) at $10 . \mathrm{m}$. Information for the longitudinal polarization scheme is shown in Figure 23 It appears that a $6^{\prime \prime}$ displacement is just adequate for this case. The beam spot distributions at the polarization collimator for the transverse scheme are shown in Figure 24 It appears that no significant degradation of polarization would occur for a 12 " displacement although the rate is limited in the case considered by a collimator smaller than the first order spot size at momenta lower than $340 \mathrm{GeV} / \mathrm{c}$. With a 6" displacement the F.W.H. M. of the spot is only slightly worse but the tails on the distribution may indicate a significant unpolarized baciground would exist. As indicated, the situation becomes worse at lower moment um.

We have obtained a cost estimate from civil engineers at Fermilab for adding pipes to the M-3 line to make a 12 "or 24" displacement possible at the momentum focus only. New pipes would be added above the present pipes from 400' to 600' and from $600^{\prime}$ to 1000 '. The rost would be approximately $\$ 50 \mathrm{~K}$. Assuming all other aspects of the present design work, this may be the cheapest way to go. Studies are continuing to determine how necessary this option is. 

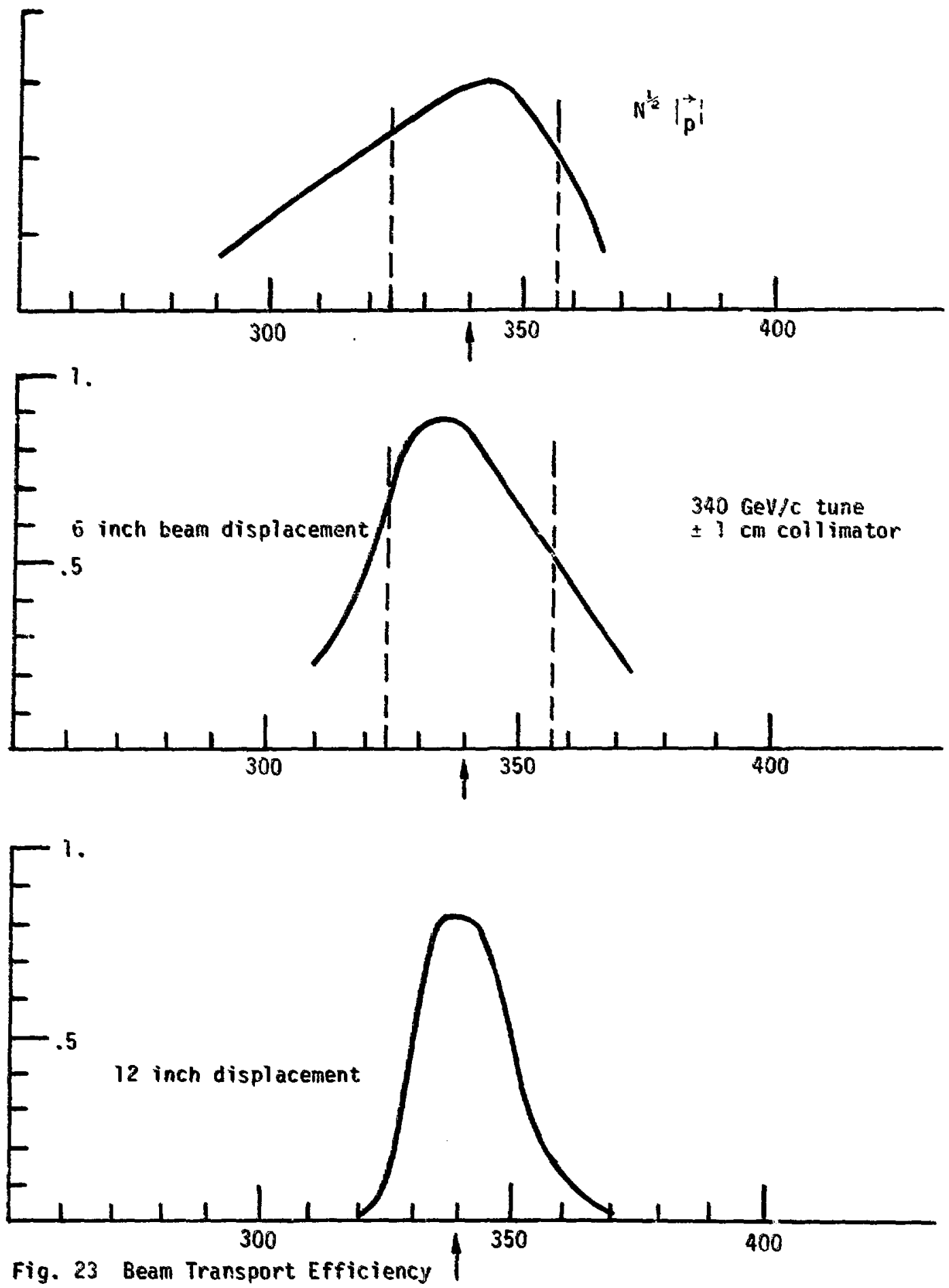


\section{$340 \mathrm{GeV} / \mathrm{C}$}
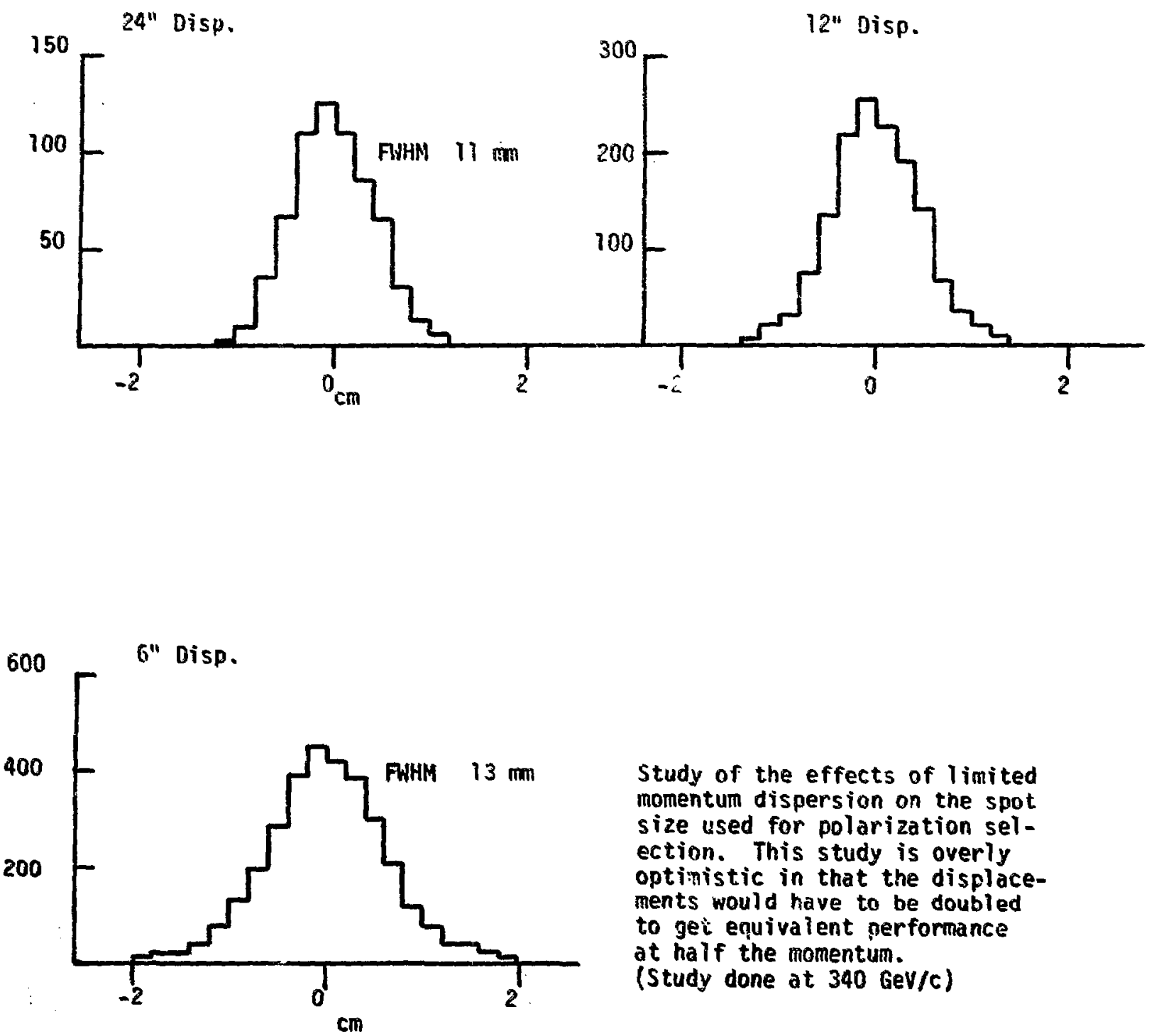

Study of the effects of limited momentum dispersion on the spot size used for polarization selection. This study is overly optinistic in that the displacements would have to be doubled to gei equivaient nerformance at half the momentum. (Study done at $340 \mathrm{GeV} / \mathrm{C}$ )

Fig. 24 Transverse Spot for Polarization Collimation 


\section{INTENSITIES}

Intensities have been calculated with a Monte Carlo program using fits to lambda and kaon production cross sections as measured by fermilab experiment $E-8$ (Table 1). Events were generated flat in $X$ and $p L^{2}$ in the production center of mass, and then weighted with the non-invariant cross section. In the laboratory frame cuts were made to select lambdas or kaons decaying between 6 . and 15 . $m$ from the production target and falling within the angular acceptance of the beam line $(1 \mathrm{~m} . \mathrm{r}$. from the beam axis).

Decays within the sweeping magnets and quadrupoles were ignored for the rate calculation. The maximum distance for accepting such decays is 1 . meter upstream into the sweeping magnet and a comparable distance downstream into the quadrupoles.

Absolute cross sections for lambda production or protons within a given momentum bite were then available to be weighted with various factors. The total lambda inclusive (including $\Sigma^{0} \rightarrow \gamma^{0}$ ) cross section for protons on Beryllium was found to be something over 14. mb. compared to a total of 270. m.b. (Figure 25) p-Be cross section.

The weights then folded in were for a $\frac{1}{2}$ interaction length target, branching ratio for $\lambda \rightarrow p \pi$ of .64 , and incident intensity of $10^{13}$ per spill. A proton momentum bite of $\pm 5 \%$ was assumed.

Similar calculations were made for $\bar{p}$ from $\vec{\Lambda}$ and for $\pi \pm$ from $k_{s}^{0}$.

The acceptance of the beam line in various configurations was found using program TURTLE in the course of a study of monentum dispersion effects.

The beam spot at the collimators for $\pi$ from $K_{S}^{0}$ is larger than the spot for $p$ or $\bar{p}$ due to the larger $p \perp$ of the decay. The collimators will cut down the $\pi$ transmission in the beam line with respect to $p$ or $\bar{p}$ transmission. The transmission ratio $\frac{\pi}{p}$ is about $\frac{2}{.3}$ at high momentum and smaller at low momentum 
with small collimator openings as may be used.

For the end point scheme all the beam entering the beaml ine may be considered polarized beam. For the transverse scheme we have to choose an optimum compromise between rate and polarization. This can range from full acceptance at the polarization collimator and no polarization to no acceptance and 64: polarization. As shown in Fig. 9, the optimization curve is rather flat from about $45 \%$ transmission and $35 \%$ polarization to about 20\% transmission and 50\% polarization. We have assumed 30\% transmission in the rate calculations but it is quite complicated to choose the actual value to use in an experiment to minimize systematic errors.

Resulting rate predictions for polarized and unpolarized beams are shown in Fig 26. The unpolarized rates can be compared to a similar CERN calculation of Fig. 27. Our rates are a factor of ten higher due to higher incident intensity and the larger momentum bite which we can transmit without depolarization. 


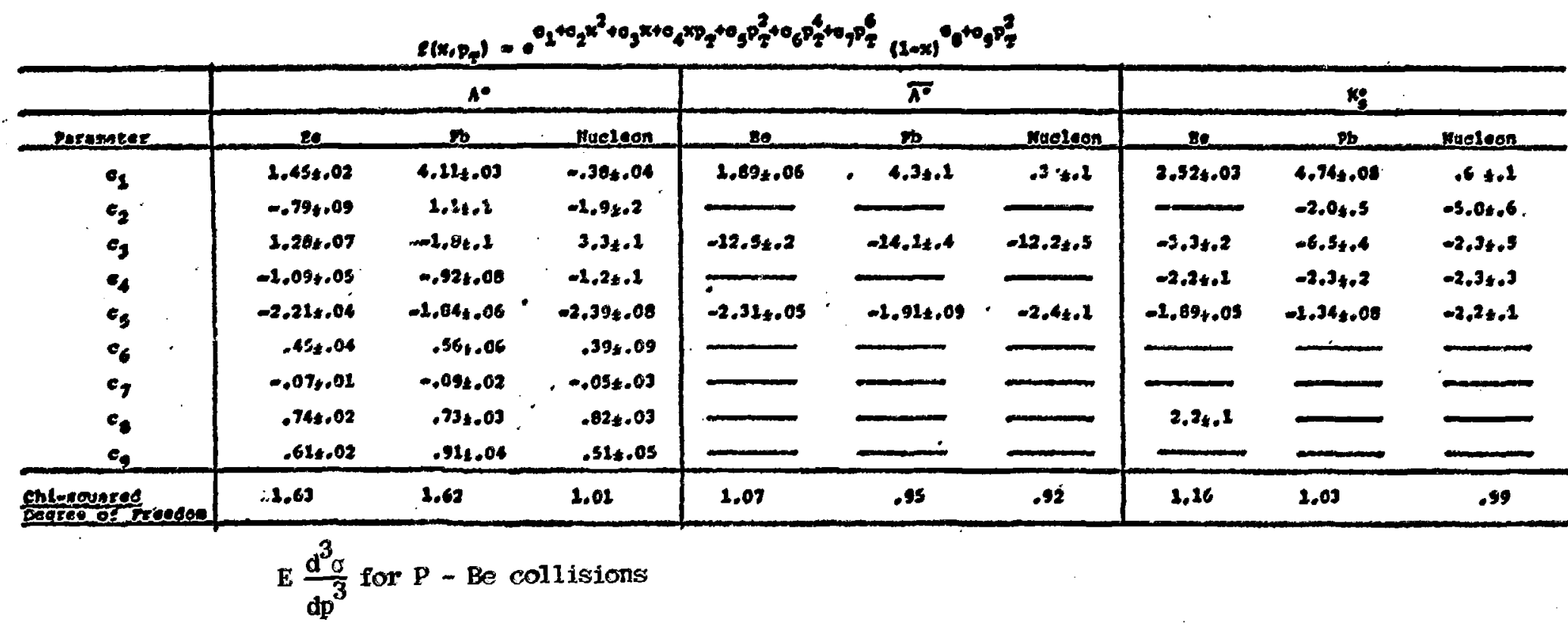

fits to E-8 Aata for $x>.2$

Table 1 Parameters obtained in fits of the Data to a Function of $x$ and $P_{T}{ }^{2}$. 
PRP

PZLAM

UF. SCALE35.

$.009292 \times x$

$.059060 \times \times \times \times \times x$

- $107865 \times \times \times \times \times \times \times \times \times \times \times$

$-156522 \times \times \times \times \times \times \times \times \times \times \times \times \times \times \times$ $.203009 \times \times \times \times \times \times \times \times \times \times \times \times \times \times \times \times \times$

$.254668 \times \times \times \times \times \times \times \times \times \times \times \times \times X \times \times \times \times X$

. 3020 ง8 $\times \times \times \times \times \times \times \times \times \times \times \times \times \times \times \times \times \times \times \times \times$ $356292 \times \times \times \times \times \times \times \times \times \times \times \times \times \times \times \times \times \times \times \times \times \times$

$.409881 \times \times \times \times \times \times \times \times \times \times \times \times \times \times \times \times \times \times \times \times \times \times \times$

$.459709 \times \times \times \times \times \times \times \times \times \times \times \times \times \times \times \times \times \times \times \times \times \times$

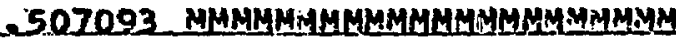

$.553955 \times \times \times \times \times \times \times \times \times \times \times \times \times \times \times \times \times \times \times \times X$

$.605035 \times \times \times \times \times \times \times \times \times \times \times \times \times \times \times \times \times \times \times$

$-652216 \quad \times \times \times \times \times \times \times \times \times \times \times \times \times \times \times \times \times \times$

$.704408 \times \times \times \times \times \times \times \times \times \times \times \times \times \times \times \times \times$

$.757097 \times \times \times \times \times \times \times \times \times \times \times \times \times \times \times$

$.90 \_613 \times \times \times \times \times \times \times \times \times \times \times \times \times$

$.853665 \times \times \times \times \times \times \times \times \times \times \times X$

$.907958 \times \times \times \times \times \times \times \times \times \times \times$

$.052057 \times \times \times \times \times \times \times \times \times$

1.20

1. 05

$-.1 .10$

Cutofi in Event

1.15

1.20

L.25

1.30

1.35

1.40

1.45

1.50

$1=55$

Cutofin in

t

Generation

1.60

1.65

JF.

SCALE 12.8 +1 3

$.5395 \times \times \times \times \times \times \times \times \times \times \times \times \times \times \times \times \times \times \times \times \times \times$

10.755.8. $\times \times \times \times \times \times \times \times$

$20.9936 \times \times \times X X$

$30.7701 \times \times \times$

40.2546 .

50. $341 \times X$

$60.3586 x$

$20.5505 x$

8.3. $92.95 x$

$90.6849 \times$

Lon.2920x

110. 333

120.9351

130.7020

140.5841

150.4134

160.2753

170.1012

280.9650

190.9500

230.8340

210.7710

220.7015

230.6540

240.5714

250.5343

260.4925

270.4067

230.3770

290.3141

300.2891

310.2607

320.2001

330.1315

-...2. 70

1.75

340,1409

1. 50

$350.113 \%$

360.890

370.645

- 2.85

380.442

1.90

1.95

... 2.00

2.05

390. 159

4002

2.12

$2 \cdot 15$

2.20

2.75

2. 30

2. 35

2.40

2.45

410.

Units are

420.

430 .

$\$+0$.

450 .

450.

470.

480.

490.

$53+1433625$

OF.

$10^{-32} \mathrm{~cm}^{2}$

Total $=14 . \mathrm{mb}$

$\sigma_{\text {Tot }}(P \mathrm{Be})=270, \mathrm{mb}$

IF.

Fig. 25 Lambda Production 


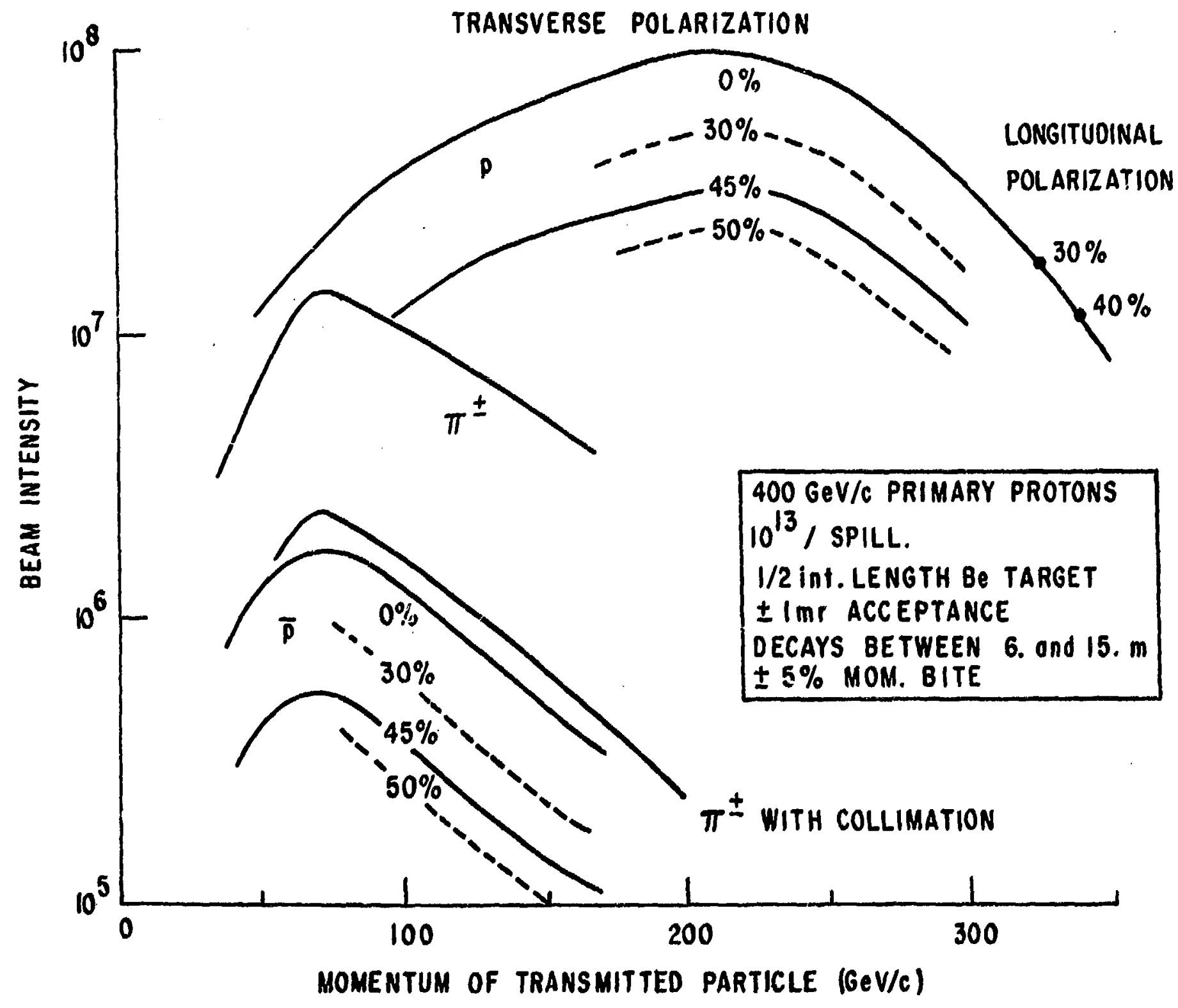

Fig. 26 Estimated Polarized Proton and Antiproton Beam-Intensities 


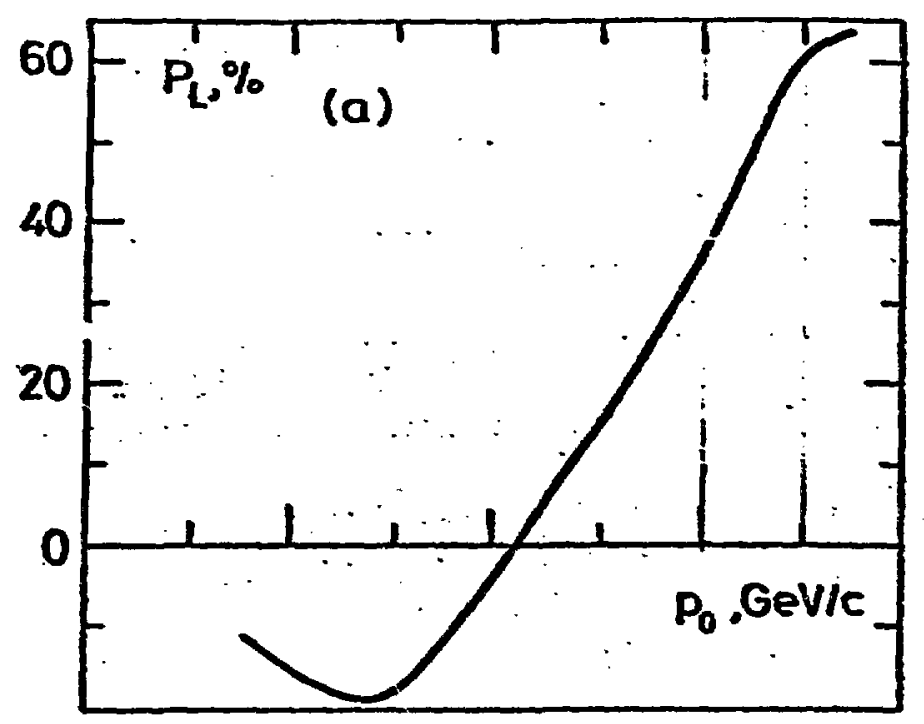

$P_{0} C=400 \mathrm{GeV}_{i} N_{\text {INC }}=3.10^{12} \mathrm{PPP}$;

$\Delta p / p= \pm 1.4 \% ; \Omega_{p}=1.6$ uster; $l(B e)=40 \mathrm{~cm}$

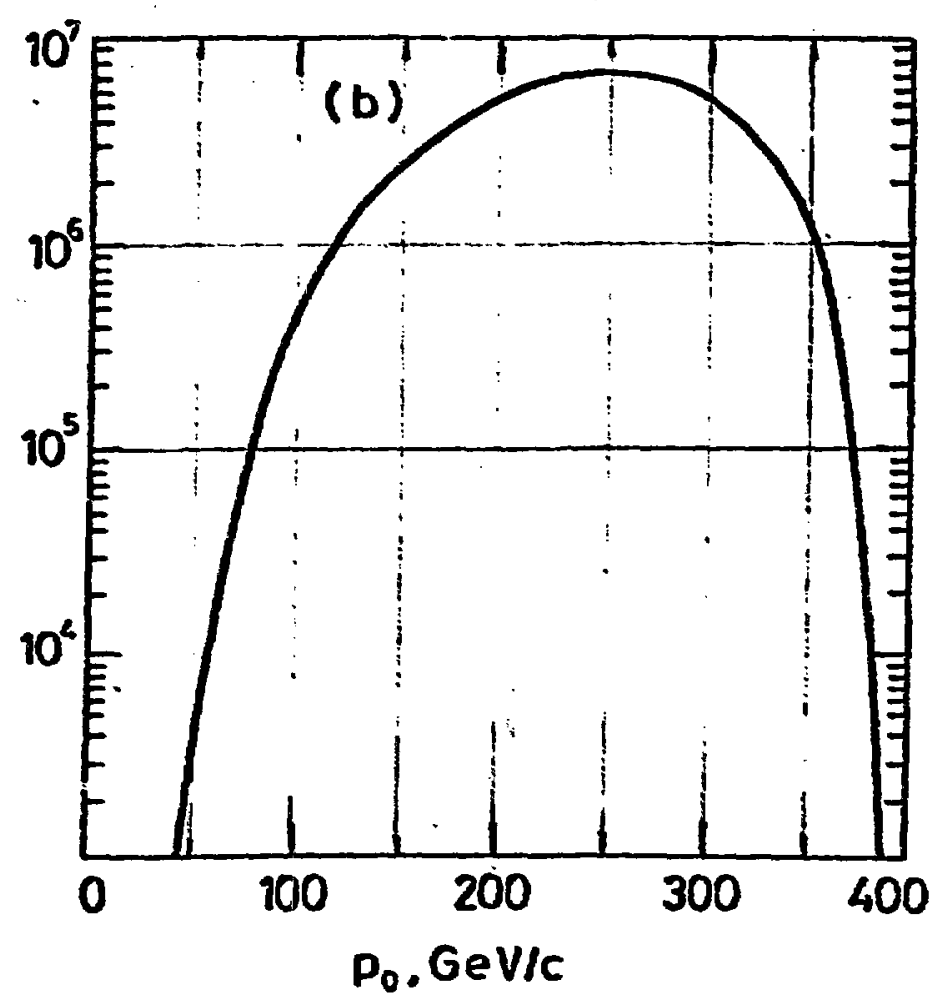

Fig. 27 CERN Proposal 


\section{TARGETING AND SWEEPING}

People have made several recommendations about the targeting and sweeping region after seeing the initial sketch of what might be needed.

i) The sweeping magnets can be drilled or cut to allow simultaneous use of the Ml, M6, and $M 3$ lines with beam splitting in meson Iab.

ii) Collimators used at high intensity should be made of aluminum so that the hadronic shower will be spread our sufficiently to allow adequate heat dissipation.

iii) The beam should be dumped far from the coils of the sweeping magnet. If the beam were dumped near to coils he rate of radiation damage might aliow us to complete one experiment but not much more.

iv) We should be compatible with other uses of the $M 2$ and $M 3$ lines, in a time sharing manner. Specifically, the high intensity $k^{0}$ experiment in $M 3$ and a fraction of the primary beam $\left(210^{10}\right.$ ) being transported directly through our sweeping system and down the M2 line.

In Figs. 28 and 29 we have sketched a targeting, sweeping and dumping scheme that incorporates these recommendations and appears to be suitable for producing the clean neutral beam necessary for the polarized beam. This is not a precise design in terms shapes of the magnet shims and collimators but shouid be good enough for the meson lab to plan around it.

Several people have asked why the second of the two sweeping magnets is needed. It is used to sweep away hadronic shower particles that leak into the collimator opening, so that they will not get into the beam line. 


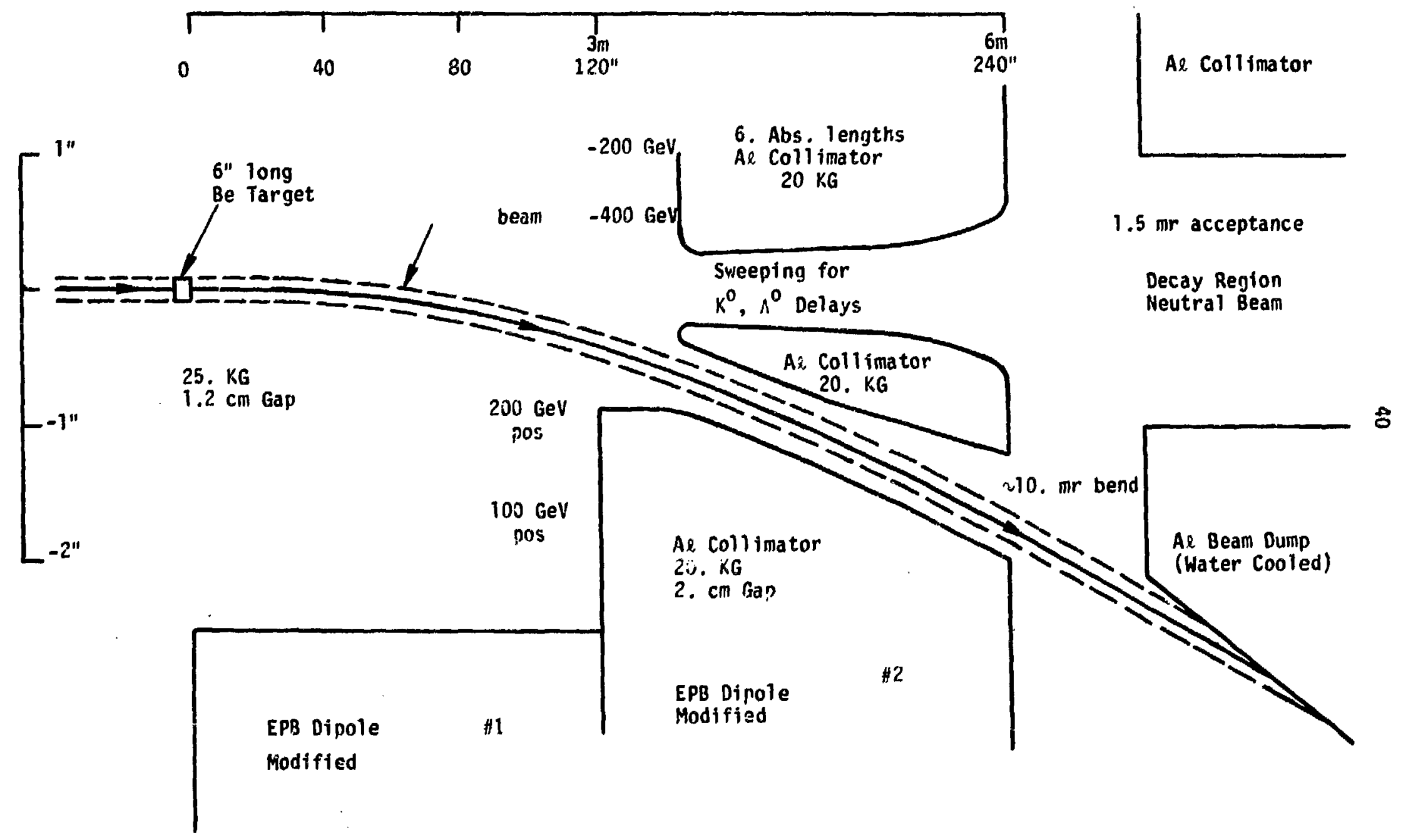

Transverse scale 1:1

Longotudinal Scale 1:40

Fig. 28 Beam Sweeping for Polarized Beam 


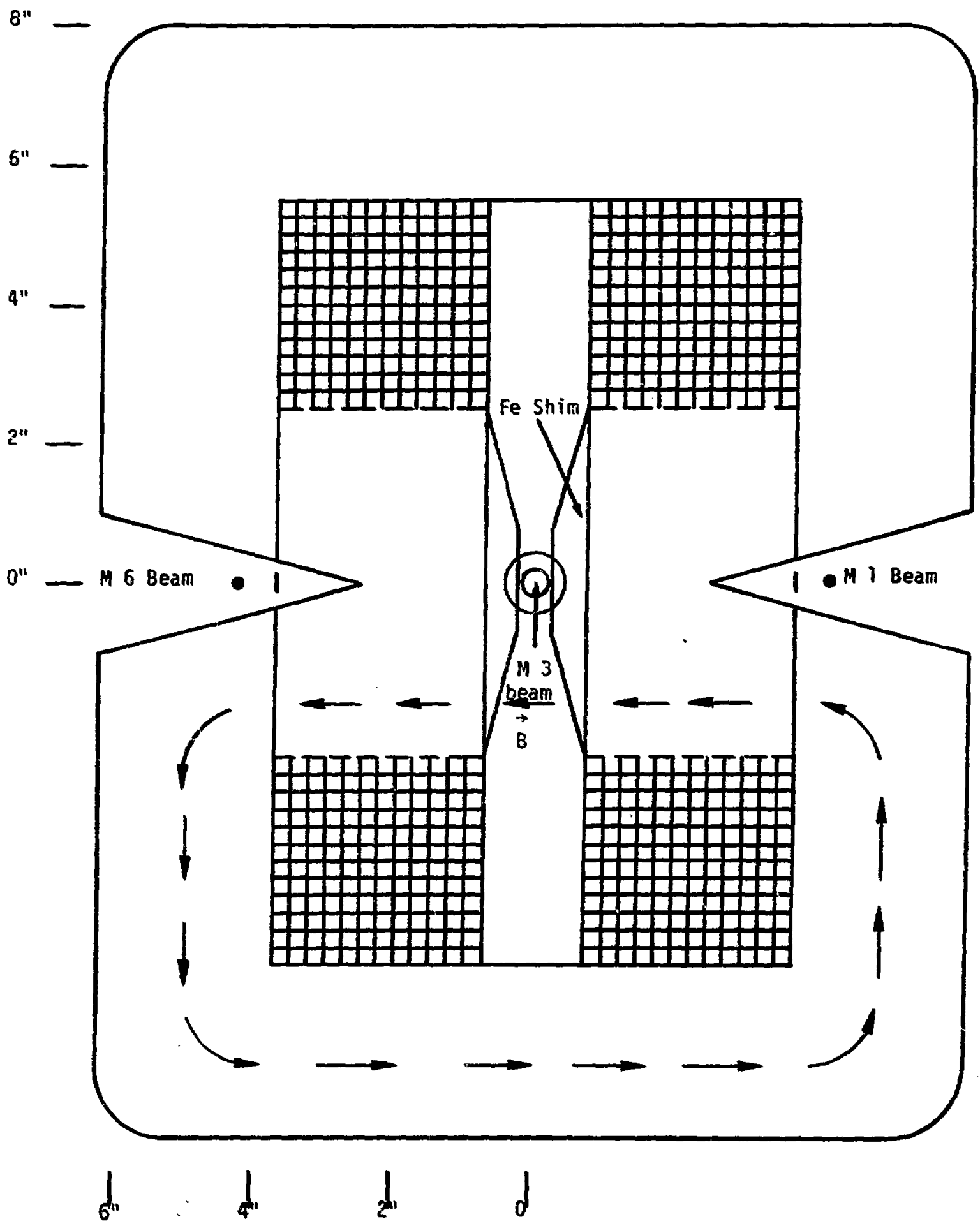

Fig. 29 Modified 5-1.5-120 EPB Dipole for Sweeping 
This may make the beam only a little cleaner at $340 \mathrm{GeV}$ but is absolutely essential at lower energies with $400 \mathrm{GeV}$ primary beam. It is unfortunate that this length of sweeping magnets is longer than the lambda decay length for energies below $100 \mathrm{GeV}$ and is a major factor in limiting beam intensity. On the other hand, for the transverse polarization scheme to work, it is essential that no decays be accepted from very close to the production target, and the sweeping magnets provide roughly the right cutoff length. The beam line can capture protons only from decays beyond the sweeping and from roughly the last meter of sweeping.

Another problem not yet fully resolved is the dumping of the neutral beam. In all designs conceived of so far, this must be done near the downstream end of the front-end hall. It was originally expected that this would be the position of the momentum focus, so that small-aperture bends could be used to bend the proton beam up above the neutral beam. In the present design the beam is rather larger at this point (roughly three inches). A design with the momentum focus near the end of the front end hall appears to be feasible in principle, but would require superconducting quadrupoles in the front end hall and would have bad depolarization properties. Bending magnets of three-inch aperature about ten feet in length can probably be accommodated in the front end hall with less difficulty than the quadrupoles required in any case.

The radiation-safety problems involved in dumping the neutral beam of roughly $10^{11}$ particles per spill for fractions of each year may be less tractable than dumping the $10^{13}$ primary beam because the front end hall is not shielded and isolated like the target box containing the target train. Apparently one would have to build an absorber which would not allow significant fractions of the hadronic shower to leak into the 
earth and contaminate the ground water. There is room for thirty feet of absorber along the beam direction so most of the problem would seem to be the transverse leakage out of three to tweive inches of steel. 


\section{MDDIFICATIONS TO MESON}

\section{A) TARCET TRAIN}

The polarized beam would require a target train with two sweeping magnets as discussed. The external bean dum abolit 7 meters from the target should not shine high momentum particles into the quadrupoles in such a way that they will be transmitted by the beam line. The primary beam at the interaction length Beryllium target mast be less than $5 . \mathrm{mm}$ in diameter for the transverse polarization scheme to work above 150. GeV/c. There is probably no point in making the beam diameter smaller than $2 . \mathrm{mm}$, particularly since heat dissipation in the beam dump would be easier to handle with a large beam spot.

We need a beam dump to dump over $10^{1}$ neutrons and gammas. This could be near 200 feet, upstream of a 3 -inch or 4-inch gap vertical bend. The neutral beam would be about 5 inches in diameter here. Alternatively, most of the neutral beam could pass through the bend and be dumped near 230 feet.

The charged beam is 3 inches in diameter at this point and can be displaced downward only 4.5 inches due to the limitations of the 12 -inch beam pipe. This displacement could be increased by oifsetting the target and M-3 beam axis upward by a few inches.

\section{B) MODIFICA TIONS TO THE M-3 LINE}

As discussed previously, it appears that the only way to get the required momentum dispersion at the collimator is to install a new beam pipe with an upward displacement of the beam at 650 feet. Pipes could be added 
most easily above the present M-3 pipe, (as opposed to below it). The pipe could go upward from the present beam height at 400 feet to about 1.5 feet above the present height at 650 feet and back down to the present height at 1000 feet. Architectural services has estimated the cost of adding such pipes at about $50 \mathrm{~K}$ dollars.

If we wish to use standard magnets (as opposed to superconducting) in the 400 foot area, the area must be extended. It must accomodate $4-4 Q$ 120 quads and two bends of ten and six feet. It appears easiest to extend this area downstream, although the beam acceptance and optics would be optimized by extending the area upstream.

If the meson beam were to be dug up extensively, we could suggest other changes to optimize the beam, assuming such changes would be less expensive as part of a large project. 


\section{OTHER CONSIDERATIONS}

If a three way split of the primary beam to meson lab is feasible with some degree of focusing of the central bean, the target train could be compatible with simultaneous use of the $M-1, M-3$, and $M-6$ lines. The $M-2$ line could operate when the M-3 line was off by shutting off the sweeping magnets. removing the neutral beam dump, and moving four magnets. Direct beam could be transmitted down the $\mathrm{M}-2$ line assuming the split could be changed from $10^{13}$ in the central beam to $10^{10}$ particles/spill.

The M-4 line could operate when a production target was used for the $M-2$ line. It may be possible to feed the M-5 line from a target for the M-6 line.

The quadrupoles for the polarized beam in the front end hall would interfere with the present positions of the $M-6$ bends and the $M-1$ quadrupoles, but with a three way split the geometry of these lines would be different.

The only significant interference with other beam lines would seem to be with the M-2 line and this seems to be at the level that could be handled in a one week down period. It might be nice to consider building the polarized beam in the M-2 line instead of the M-3 line but this would involve severe modifications to the beam and pipes as well as such unpleasantries as horizontal sweeping on the target train with the primary beam dump shining muons down the $M-l$ line and $M-6$ line. 


\section{Magnets Needed \\ (For Optimum Design)}

Sweeping

Two (5-1. 5-120) EPB Dipoles

First Quads

Roughiy 40 feet of 3 inch quads with approximately 12. KG pole tip field. Attanged as a triplet.

Bencs for Dumping

4 bends of at least $50, k G-m$ (one of these would have to have

a 4 inch aperture and the others 3 inch apertures).

Second Set of Quads

Roughiy 40 feet of 4 inch quads with 12. $k G$ pole tip field.

Arranged as a triplet.

Momentum Selection Bends

4 bends of at least 50, kG-m (all with 4 inch aperture).

Verniers

2 bends of roughly 4 . kG-m with 4 inch aperture.

Third Set of Quads

(Same as second set)

Final set of quads

(Same as First Set) 


\section{SPIN PRECESSION MAGNETS}

A universal set of spin precession magnets would be eight magnets of at least $27,4 \mathrm{KG}-\mathrm{m}$ field integral which fit into roughly $16 . \mathrm{m}$ and have 5 . inch gaps. The power supplies could be ramped and the magnet polarities switched on a spill to spill bases. The magnets would probab?y have to be Iaminated.

For most experiments this full set would not be necessary but the minimum set for any experiment is four magnets with 3. inch gaps.

\section{APPROXIMATE MAGNET LIST}

\begin{tabular}{|c|c|c|}
\hline$z($ feet $)$ & No. & Magnets \\
\hline 0 & 1 & $5-1.5-120$ \\
\hline 11 & $\mathbf{i}$ & $5-1.5-120$ \\
\hline 52 & 4 & $3 \mathrm{Q} 120 \mathrm{II}$ \\
\hline 105 & 1 & $6-3-120$ \\
\hline 180 & 2 & Vernier Bends \\
\hline 208 & , & $6-3=120$ \\
\hline 255 & 1 & $6-3-120$ \\
\hline 365 & 1 & $10-I V-72$ or $6-3-120$ \\
\hline 376 & 4 & $4-Q-120 \mathrm{II}$ \\
\hline 420 & 1 & $10-I V-72$ \\
\hline 650 & 1 & $16-I V-72$ or $6-3-120$ \\
\hline 680 & 1 & $10-I V-72$ or $6-3-120$ \\
\hline 1005 & 1 & $10-I V=72$ \\
\hline 1012 & 4 & $4-Q-120 I I$ \\
\hline 1350 & 4 & $3-Q-120 I I$ \\
\hline 1405 & 4 or 8 & $18-V I-72$ or $10-I V-72$ \\
\hline
\end{tabular}




\section{REFERENCES}

1. Measurement of the Decay Parameters of the $\Lambda^{\circ}$ Particle, J. W. Cronin and O. E. Overseth, Phys. Rev. 129, 1795, (1963).

2. Ea rlier work on this subject includes:

i) O. E. Overseth, NAL 1969 Summer Study Report, SS-118, Vol. I.

ii) O. E. Overseth and J. Sandweiss, NAL 1969 Summer Study Report SS-120, Vol, I.

3. Proceedings of the Symposium on Experiments Using Enriched Antiproton, Polarized Proton, and Polarized Antiproton Beams at Fermilab energies, edited by A. Yokosawa, ANL-HEP-CP-77-45 (1977).

4. On the Study of Spin Effects at SPS Energies by Making use of a Polarized Beam Facility, Letter of Intent, CERN/SPSC/76-95, SPSC/I 87, 11 November, 1976.

5. Study of Spin Effects at SPS Energies using a Polarized Proton Beam, CERN Proposal, CERN/SPSC/77-61, SPSC/p. 87, July 19, 1977.

6. High Energy Polarized Proton Beam from $\AA^{\circ}$ Decay, P. Dalpiaz, J. A. Jansen and G. Coignet, GERN/ECFA/72/4, Vol. 1, p. 284 .

7. Precession of the Polarization of Particles Moving in a Homogeneous Electromagnetic Field, V. Bargmann, L. Michel, and V. L. Telegdi, Phys. Rev. Lett. 2, p. 435 (1959).

8. Spin Precession in a Quadrupole Lens, S. Nurushev, CERN preprint submitted to Nuclear Instruments and Methods.

9. A Method of Measuring the Polarization of High Momentum Proton Beams, David G. Underwood, ANL-HEP-PR-77-56. 
10. J. Roberts - In the Proceedings of the Michigan Conference on Pola rized Beams, October, 1977.

11. Fea sibility Study on the Designs of Short Lived Beams in the Proton Central Experimental Area, D. P. Eartly, Fermilab TM-546 6029, April, 1975.

12. P-West High Intensity Secondary Beam Area Design Report, Fermilab, December 1976. 


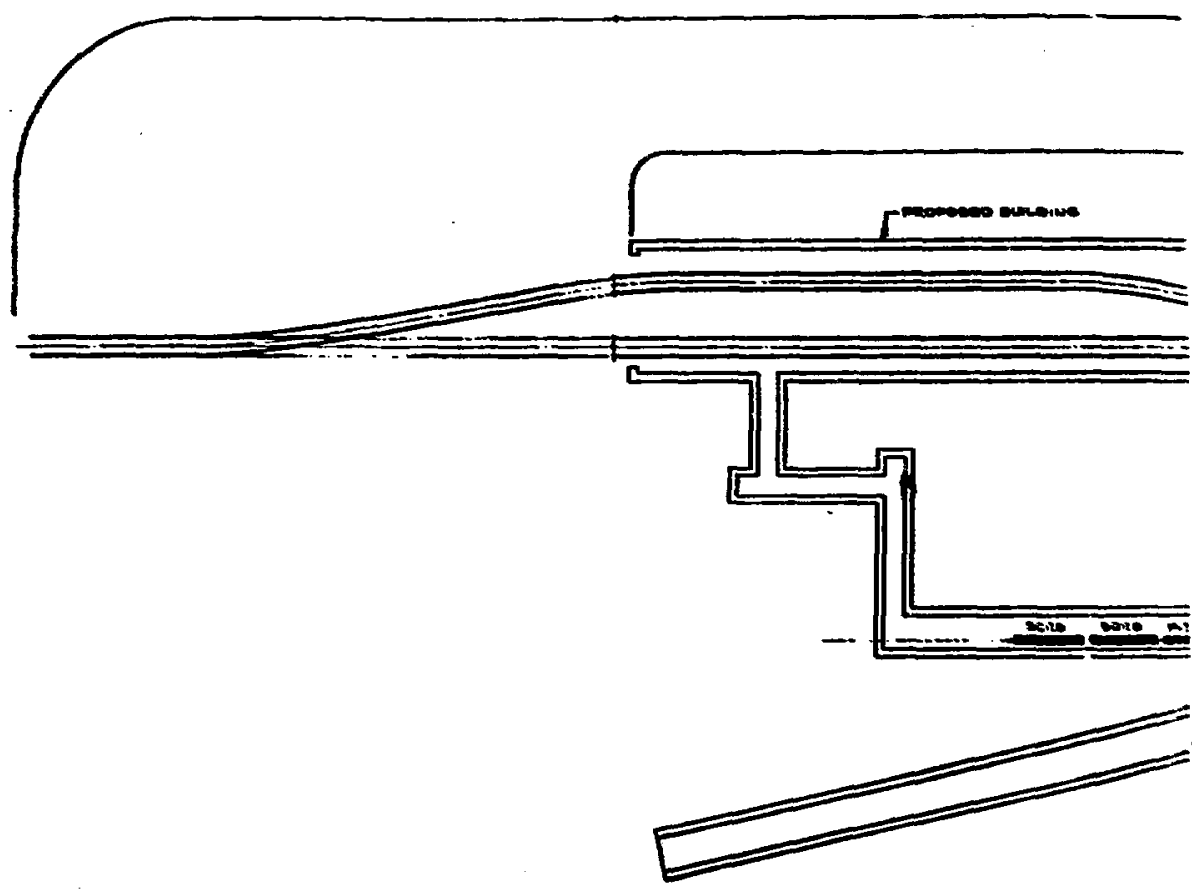

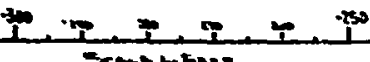




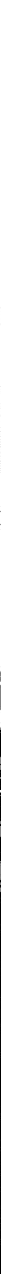




\section{Tremse}

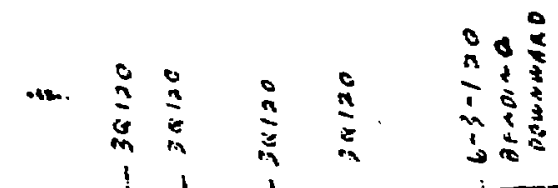

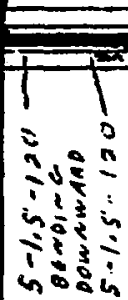

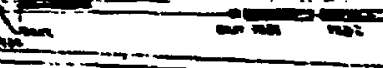




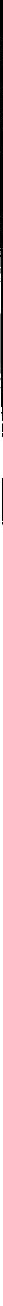



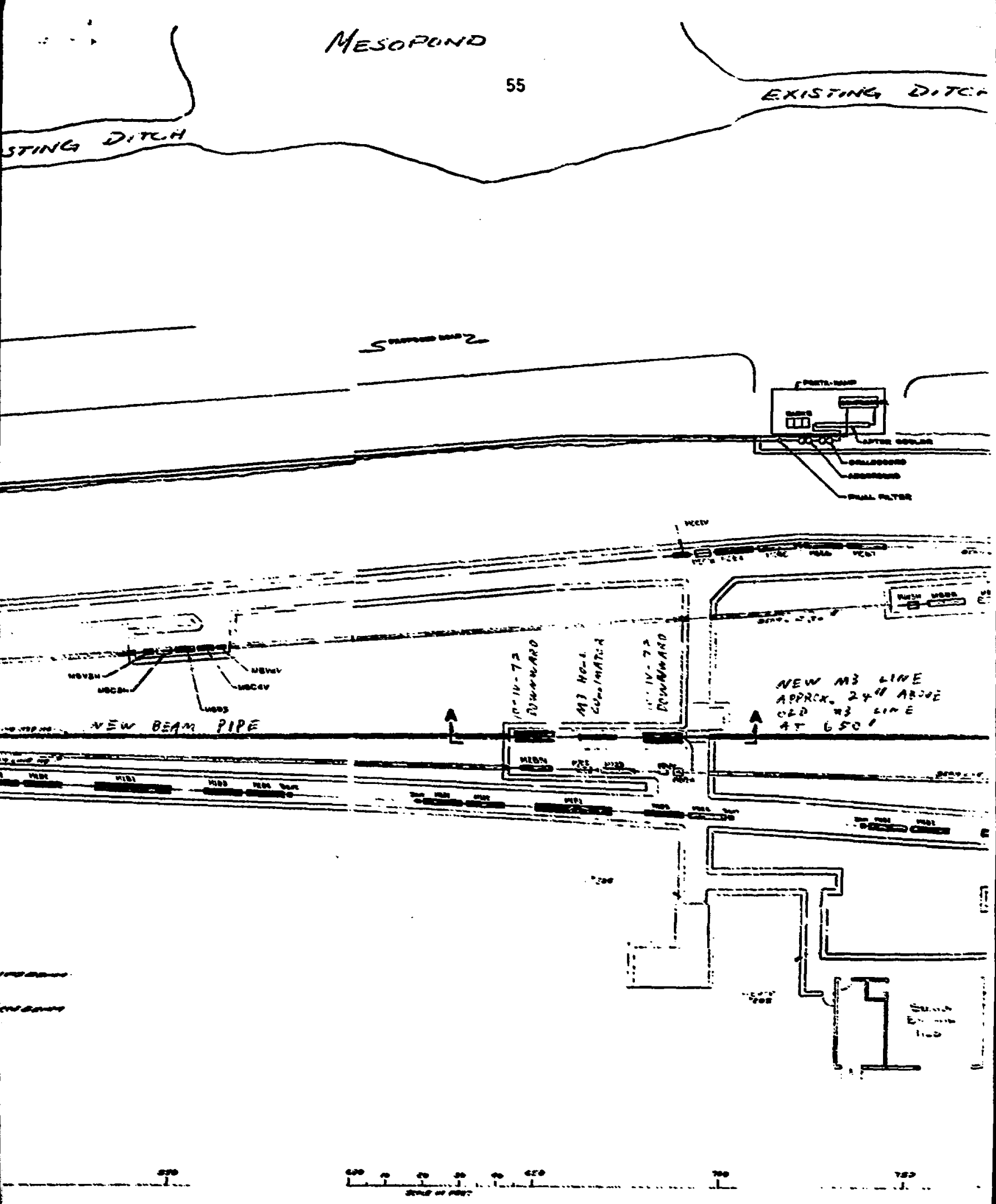


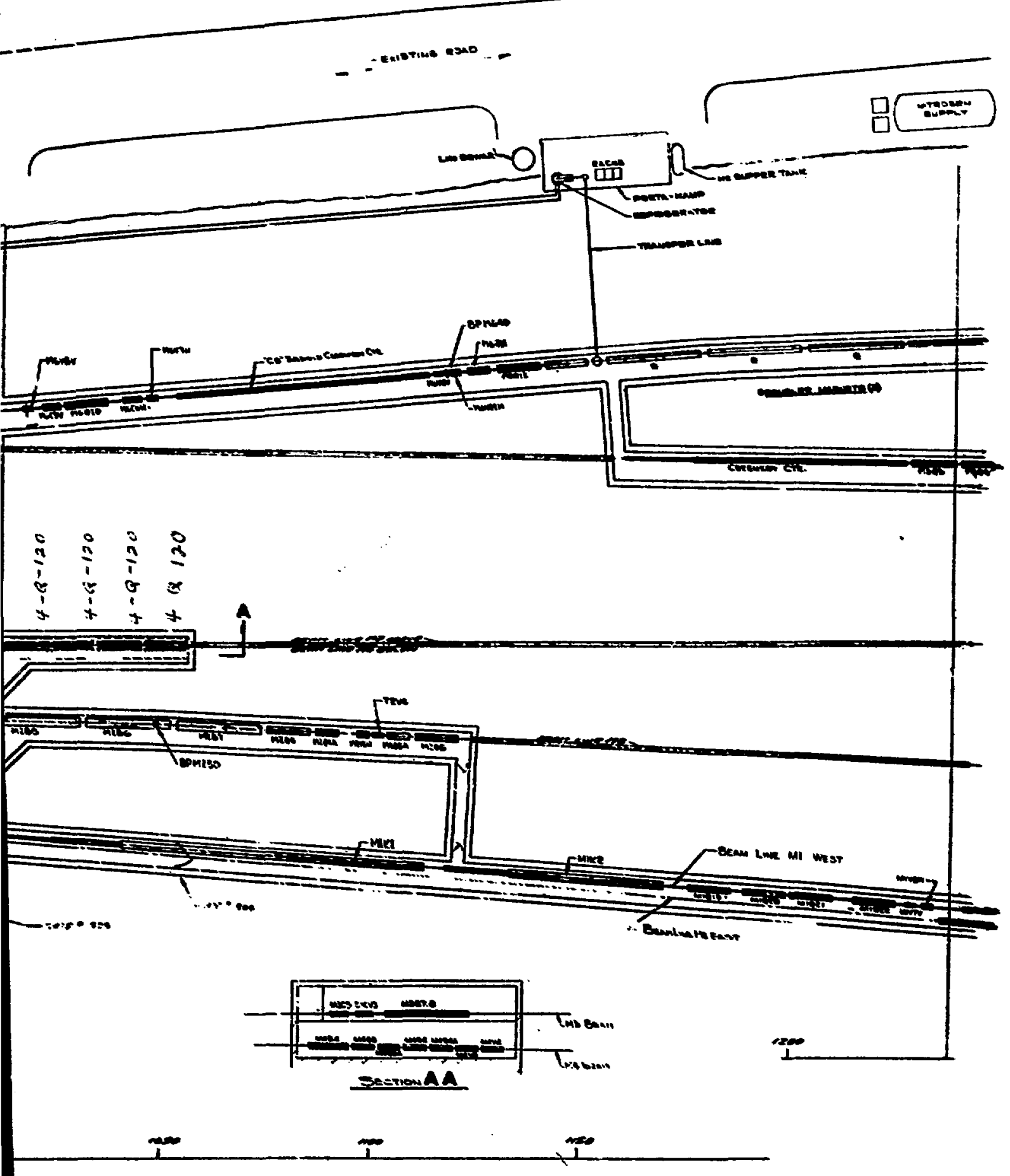


ॠ๐+人

5
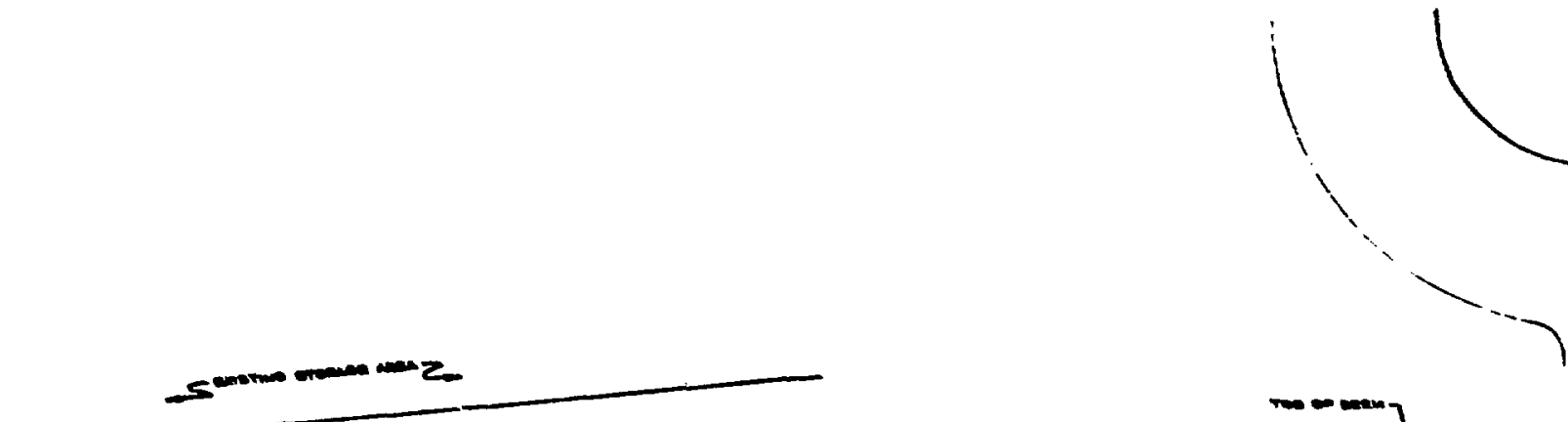

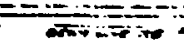
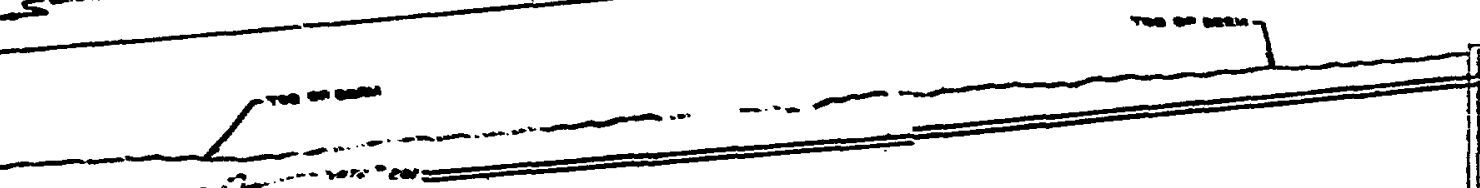

$=$ $=$

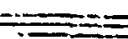

\section{S $=m \cdot \operatorname{sen}$}

NEW

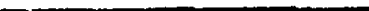
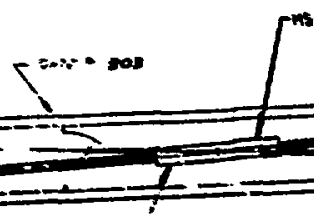

nsthecomendid

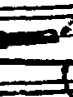
$=$

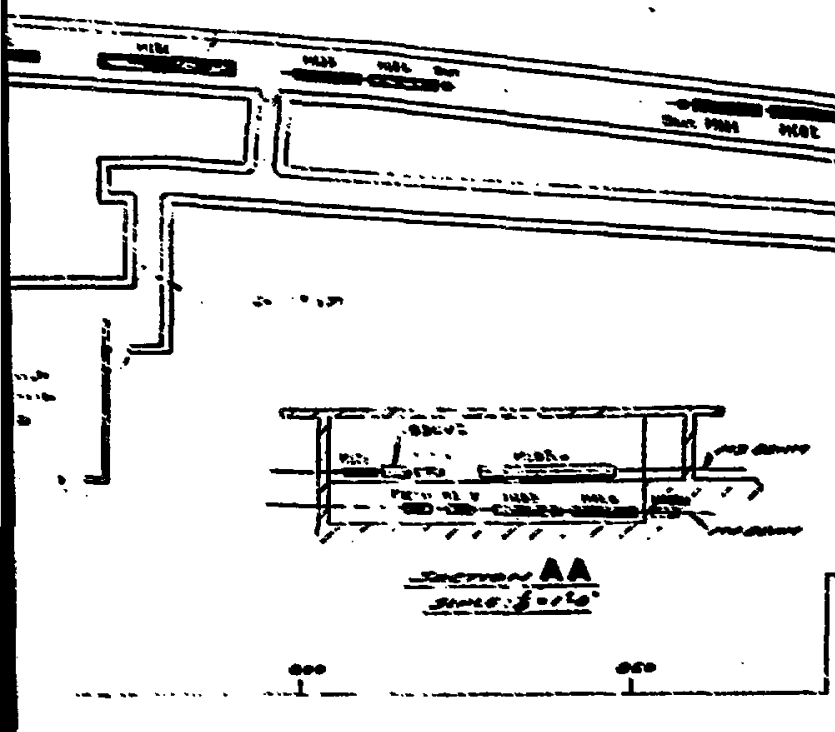

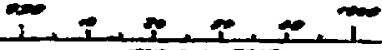

